Vehicle System Dynamics: International J ournal of Vehicle Mechanics and Mobility,

Volume 53, Issue 11, 2015, pp1555-1579

DOI: $10.1080 / 00423114.2015 .1064972$

\title{
Rear Wheel Torque Vectoring Model Predictive Control with Velocity Regulation for Electric Vehicles
}

\author{
Efstathios Siampis*, Efstathios Velenis and Stefano Longo \\ Centre for Automotive Engineering, School of Aerospace, Transport and Manufacturing, \\ Cranfield University, Cranfield, UK
}

(Received 00 Month 20XX; accepted 00 Month 20XX)

\begin{abstract}
In this paper we propose a constrained optimal control architecture for combined velocity, yaw and sideslip regulation for stabilization of the vehicle near the limit of lateral acceleration using the rear axle electric torque vectoring configuration of an electric vehicle. A nonlinear vehicle and tyre model are used to find reference steady-state cornering conditions and design two Model Predictive Control (MPC) strategies of different levels of fidelity: one that uses a linearized version of the full vehicle model with the rear wheels' torques as the input, and another one that neglects the wheel dynamics and uses the rear wheels' slips as the input instead. After analysing the relative trade-offs between performance and computational effort, we compare the two MPC strategies against each other and against an unconstrained optimal control strategy in Simulink and Carsim environment.
\end{abstract}

Keywords: torque vectoring; model predictive control; combined velocity, yaw and sideslip control; limit-handling

\section{Notation}

$\begin{array}{ll}\alpha & \text { Tyre slip angle } \\ \beta & \text { Vehicle sideslip angle at its centre of mass } \\ \delta & \text { Steering angle } \\ \epsilon & \text { Slack variable } \\ \mu_{x}, \mu_{y} & \text { Longitudinal and lateral tyre force coefficient } \\ \mu_{\max } & \text { Tyre/road friction coefficient } \\ \psi & \text { Vehicle yaw angle at its centre of mass } \\ \omega & \text { Wheel angular rate } \\ a_{x}, a_{y} & \text { Vehicle longitudinal and lateral acceleration at its centre of mass } \\ f_{x}, f_{y}, f_{z} & \text { Longitudinal, lateral and normal tyre force } \\ g & \text { Constant of gravitational acceleration } \\ i, j & \text { Subscripts } i=F, R \text { (front, rear), } j=L, R \text { (left, right) } \\ \ell_{F}, \ell_{R} & \text { Longitudinal distance of centre of mass from the front and the rear track } \\ m & \text { Mass of the vehicle } \\ r & \text { Wheel radius } \\ s, s_{x}, s_{y} & \text { Total, longitudinal and lateral slip }\end{array}$

*Corresponding author. Email: e.siampis@cranfield.ac.uk

Published by Taylor and Francis. This is the Author Accepted M anuscript issued with: Creative Commons Attribution Non-Commercial License (CC:BY:NC 3.0). 
$w_{L}, w_{R} \quad$ Lateral distance of centre of mass from the left and right wheels

$B \quad$ Pacejka's Magic Formula stiffness factor

$C \quad$ Pacejka's Magic Formula shape factor

$D \quad$ Pacejka's Magic Formula peak value

$I_{w} \quad$ Wheel moment of inertia of each wheel about its axis of rotation

$I_{z} \quad$ Vehicle moment of inertia

$N \quad$ Horizon

$N_{p} \quad$ Prediction horizon

$N_{u} \quad$ Control horizon

$R \quad$ Vehicle path radius

$T \quad$ Torque

$T_{s} \quad$ Sampling time

$T_{\text {sim }} \quad$ Simulation time

$V \quad$ Vehicle velocity at its centre of mass

\section{Introduction}

In the past few years it has been recognised that active control of the vehicle's velocity is not only a very effective strategy in the limits of lateral acceleration but also crucial in cases of terminal understeer behaviour [22]. The necessity for velocity regulation in such cases is already mentioned by van Zanten et al. [24] who points out that especially in the case of J-turns, where the turning radius is reduced continuously along the trajectory (a scenario typical on highway exits), the Electronic Stability Control (ESC) yaw moment correction on the lateral dynamics alone is not sufficient. This early remark on the importance of longitudinal control was later realised as one of the ESC new functions in [17], where correction of terminal understeer behaviour is achieved by superimposing individual braking of all four wheels on the standard ESC intervention. Similar solutions applied on a Four Wheel Drive (4WD) Electic Vehicle (EV) can be found in $[15,16]$. In [15] the torque request from the driver is reduced when the lateral acceleration exceeds a specific threshold, while in [16] a velocity limit is set as a function of the desired yaw rate. In [12] a controller providing decoupled longitudinal force and yaw moment inputs at the higher level is combined with a static control allocation scheme to calculate forces and actuator inputs. In [22] a multivariable control architecture to address velocity, yaw and sideslip regulation in terminal understeer is presented. Simulation results using a driver model in a U-turn scenario show that the controller not only keeps the vehicle within the road boundaries, but also allows for a smoother negotiation of the corner with less steering effort from the driver.

In this paper we propose a constrained optimal control architecture that stabilizes the vehicle near the limits of lateral acceleration while accounting for the important in such cases system constraints. MPC, a control strategy tracing its origins in the chemical processes industry [19], has been increasingly popular in the industry and academia due to its ability to naturally handle multivariable system constraints. Looking in the automotive active system applications, a variety of MPC solutions can be found. For example, in [4] we find a Linear Time Varying MPC (LTV-MPC) strategy for controlling the lateral dynamics of the vehicle using independent braking of the four wheels. Simulation results show that the LTV-MPC controller successfully completes the sine and dwell test but with a considerable decrease in speed due to the braking strategy 
used. An example of an MPC application for active lateral dynamics control utilizing the steer-by-wire system of a Rear Wheel Drive prototype vehicle can be found in [5]. For the MPC formulation velocity dependent bounds are imposed on the yaw rate and sideslip angle in a way similar to the envelope control concept from the aerospace industry. Simulation and experimental results using a slalom manoeuvre show that the controller restricts the steering command from the driver when the envelope bounds are violated. In [7] two explicit MPC formulations for a yaw stability controller using Active Front Steering (AFS) and differential wheel braking are presented. Experimental results using the less computationally expensive switched MPC strategy show that the controller can successfully stabilize the vehicle in a fast double lane change on a slippery road by constraining the tyre slip angles within their limits. Another example of an explicit MPC law can be found in [6], where a yaw control strategy using a rear active differential is presented. Here the Nonlinear MPC (NMPC) problem is solved offline using the nearest point approach. Simulation results show a good agreement between the proposed approach and a nominal NMPC controller but with some chattering, which could be potentially corrected with higher number of offline computed points but at the expense of higher memory and computational requirements. A different approach can be found in [10], where an MPC strategy for roadway departure prevention using AFS and braking utilizes future road information and a driver model to ensure that the current vehicle state belongs to the set of states that will evolve to a desired final set [8]. For the MPC formulation the cost function penalizes only the control effort, while the road boundaries are set as constraints. Simulation tests show that the controller can successfully keep the vehicle within the lane boundaries in the case of overspeeding through a curve.

In this work we propose an MPC strategy for combined velocity, yaw and sideslip regulation for stabilization of the vehicle near the limits of lateral acceleration using the rear axle electric torque vectoring configuration of an electric vehicle. While using an MPC strategy has obvious advantages for the specific application at hand, it also has distinct disadvantages, which are mainly connected to the computational time needed to construct and solve the resulting optimization problem. Based on this observation, the goal of this paper is not only to develop an appropriate MPC strategy for the demanding task of stabilizing the vehicle near the limits of handling in the best possible way, but also one that can be implemented in real time. The structure of the paper is as follows: after introducing the nonlinear vehicle and tyre models along with the steady-state cornering analysis used to generate the reference for the controller to follow, the basic MPC problem is explained. Since the main disadvantage of using an MPC strategy is the computational effort attached to it, two MPC strategies of different complexity are then constructed: 1) one using the full vehicle model and 2) a simpler one that neglects the wheel speed dynamics. The effect of varying the sampling time and the horizon on the performance and the computational load of each strategy are then analysed. Finally, the two strategies are compared against each other in Simulink environment and against a Linear Quadratic Regulator (LQR) strategy [22] in CarSim environment.

\section{Vehicle Model and Reference Generation}

In this section we introduce the vehicle and tyre models used in this paper. The formulation is similar to the one found in $[22,26]$, where the interested reader can refer to for more details. 


\subsection{Vehicle Model}

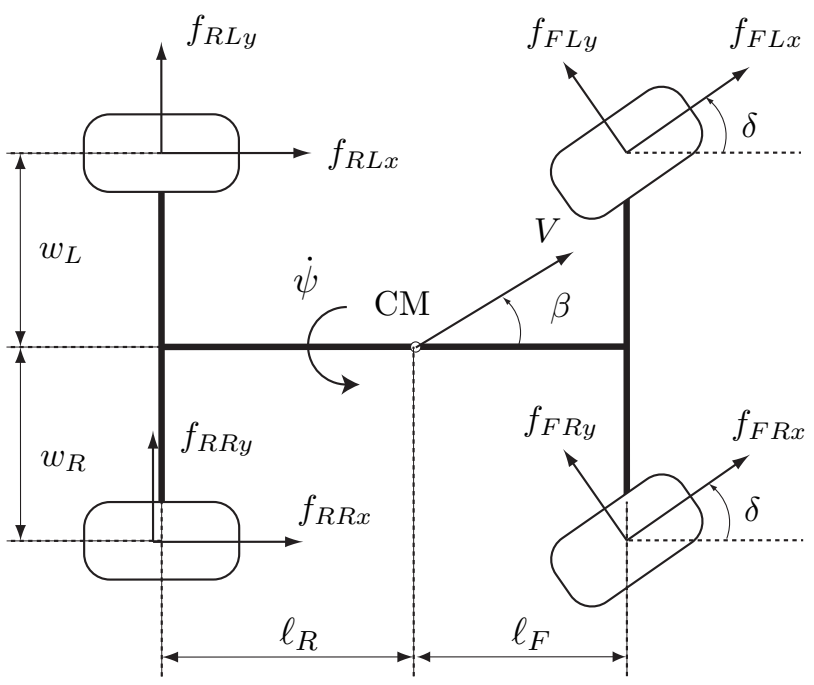

Figure 1. The four-wheel vehicle model.

The Equations Of Motion (EOM) for the four-wheel vehicle model with front wheel steering (Fig. 1) are

$$
\begin{aligned}
m \dot{V} & =\left(f_{F L x}+f_{F R x}\right) \cos (\delta-\beta)-\left(f_{F L y}+f_{F R y}\right) \sin (\delta-\beta) \\
& +\left(f_{R L x}+f_{R R x}\right) \cos \beta+\left(f_{R L y}+f_{R R y}\right) \sin \beta \\
\dot{\beta} & =\frac{1}{m V}\left[\left(f_{F L x}+f_{F R x}\right) \sin (\delta-\beta)+\left(f_{F L y}+f_{F R y}\right) \cos (\delta-\beta)\right. \\
& \left.-\left(f_{R L x}+f_{R R x}\right) \sin \beta+\left(f_{R L y}+f_{R R y}\right) \cos \beta\right]-\dot{\psi}, \\
I_{z} \ddot{\psi} & =\ell_{F}\left[\left(f_{F L y}+f_{F R y}\right) \cos \delta+\left(f_{F L x}+f_{F R x}\right) \sin \delta\right]-\ell_{R}\left(f_{R L y}+f_{R R y}\right) \\
& +w_{L}\left(f_{F L y} \sin \delta-f_{F L x} \cos \delta-f_{R L x}\right)+w_{R}\left(f_{F R x} \cos \delta-f_{F R y} \sin \delta+f_{R R x}\right) \\
I_{w} \dot{\omega}_{i j} & =T_{i j}-f_{i j x} r, \quad i=F, R, j=L, R .
\end{aligned}
$$

where the relevant variables and parameters are as defined under the Notation section at the beginning of the paper.

The tyre forces $f_{i j x}$ and $f_{i j y}$ in the above EOM are found as functions of the tyre slip using Pacejka's Magic Formula (MF) [3]. In particular, we obtain the resultant tyre force coefficient as a function of the resultant slip at each tyre from the MF:

$$
\mu_{i j}\left(s_{i j}\right)=\operatorname{MF}\left(s_{i j}\right)=D \sin \left(C \operatorname{atan}\left(B s_{i j}\right)\right)
$$

where $s_{i j}=\sqrt{s_{i j x}^{2}+s_{i j y}^{2}}$ is the resultant tyre slip with $s_{i j x}$ and $s_{i j y}$ the theoretical longitudinal and lateral slip quantities respectively [3], and $D=\mu_{\max }$ is the tyre/road friction coefficient. Then using the friction circle equations

$$
\mu_{i j x}=-\frac{s_{i j x}}{s_{i j}} \mu_{i j}\left(s_{i j}\right), \quad \mu_{i j y}=-\frac{s_{i j y}}{s_{i j}} \mu_{i j}\left(s_{i j}\right)
$$


we obtain the tyre force coefficients $\mu_{i j x}$ and $\mu_{i j y}$ in the longitudinal and lateral direction.

Neglecting the pitch and roll rotation along with the vertical motion of the sprung mass of the vehicle, the vertical force $f_{i j z}$ on each of the four wheels can be calculated using the static load distribution and the longitudinal/lateral weight transfers under longitudinal/lateral acceleration [26]. The longitudinal and lateral tyre forces are then given by

$$
f_{i j x}=\mu_{i j x} f_{i j z}, \quad f_{i j y}=\mu_{i j y} f_{i j z} .
$$

The values for the above vehicle and tyre parameters used in this paper correspond to a compact family car and can be found in Table 1 .

\begin{tabular}{cccc} 
Table 1. & Vehicle and tyre parameters. \\
\hline Parameter & Value & Parameter & Value \\
\hline$m(\mathrm{~kg})$ & 1420 & $\ell_{F}(\mathrm{~m})$ & 1.01 \\
$I_{z}\left(\mathrm{kgm}^{2}\right)$ & 1027.8 & $\ell_{R}(\mathrm{~m})$ & 1.452 \\
$I_{w}\left(\mathrm{kgm}^{2}\right)$ & 0.6 & $r(\mathrm{~m})$ & 0.3 \\
$w_{L}(\mathrm{~m})$ & 0.81 & $B$ & 24 \\
$w_{R}(\mathrm{~m})$ & 0.81 & $C$ & 1.5 \\
$h(\mathrm{~m})$ & 0.55 & $D$ & 0.9
\end{tabular}

\subsection{Reference Generation}

In order to derive feasible targets for the controller to follow, steady-state cornering analysis of the four-wheel vehicle model (1) is used. Neglecting the wheel speed dynamics and enforcing the steady-state cornering conditions

$$
\dot{V}=0, \quad \dot{\beta}=0, \quad \ddot{\psi}=0,
$$

we obtain three algebraic equations with six unknowns, namely the equilibrium state $\left(V^{\mathrm{ss}}, \beta^{\mathrm{ss}}, R^{\mathrm{ss}}=V^{\mathrm{ss}} / \dot{\psi}^{\mathrm{ss}}\right)$ and input $\left(\delta^{\mathrm{ss}}, s_{R L x}^{\mathrm{ss}}, s_{R R x}^{\mathrm{ss}}\right)$. Providing three of the six unknowns (in this work the triplet $\left(V^{\mathrm{ss}}, R^{\mathrm{ss}}, \delta^{\mathrm{ss}}\right)$ ), we can then solve the steady-state equations using the fsolve function in MATLAB (a nonlinear equation solver) to obtain the rest of the unknowns.

Based on the above steady-state analysis, we next examine the feasibility of the vehicle path radius as requested by the driver. Similar to common practice in vehicle stability control [21] we obtain an estimate of the driver's intended path using a neutral steer linear bicycle model under steady-state cornering. We therefore set

$$
R_{k i n}=\left(\ell_{F}+\ell_{R}\right) / \tan \left(\delta^{\mathrm{ss}}\right) .
$$

The desired path radius may or may not be feasible depending on the vehicle's velocity. Consider for example the steady-state conditions for a fixed $\delta^{\text {ss }}$ and a range of $V^{\text {ss }}$ in Fig. 2. In all three cases the desired $R^{\mathrm{ss}}=R_{k i n}$ is around $14 \mathrm{~m}$, according to the steering command of $\delta^{\mathrm{ss}}=10 \mathrm{deg}$. Then, for a vehicle velocity of $V^{\mathrm{ss}}=10.75 \mathrm{~m} / \mathrm{s}$ (green curve) the vertical red dashed line corresponding to $R_{k i n}$ intersects the curve of the calculated steady-state conditions, hence the requested $R_{\text {kin }}$ is feasible. On the other hand, if the vehicle velocity is $V^{\mathrm{ss}}=11.25 \mathrm{~m} / \mathrm{s}$ (purple curve) the $R_{k i n}$ is smaller than the minimum achievable $R^{\text {ss }}$ and not longer feasible. In this case the controller will select a steady-state condition such that the desired $R_{k i n}$ becomes feasible by reducing the 
vehicle velocity. Taking into consideration the driver's intention, this velocity reduction will have to be minimal. To this end we select a steady-state velocity such that $R_{k i n}$ coincides with the minimum $R^{\mathrm{ss}}$, which in the above example corresponds to a vehicle velocity of $V^{\text {ss }}=11 \mathrm{~m} / \mathrm{s}$ (blue line).

In summary, the target steady-state condition for the controller to follow is determined using the current steering command from the driver $\left(\delta^{\mathrm{ss}}\right)$, the corresponding kinematic radius $\left(R^{\mathrm{ss}}=R_{k i n}\right)$, and either the current or maximum vehicle velocity $\left(V^{\mathrm{ss}}\right)$ as discussed above.

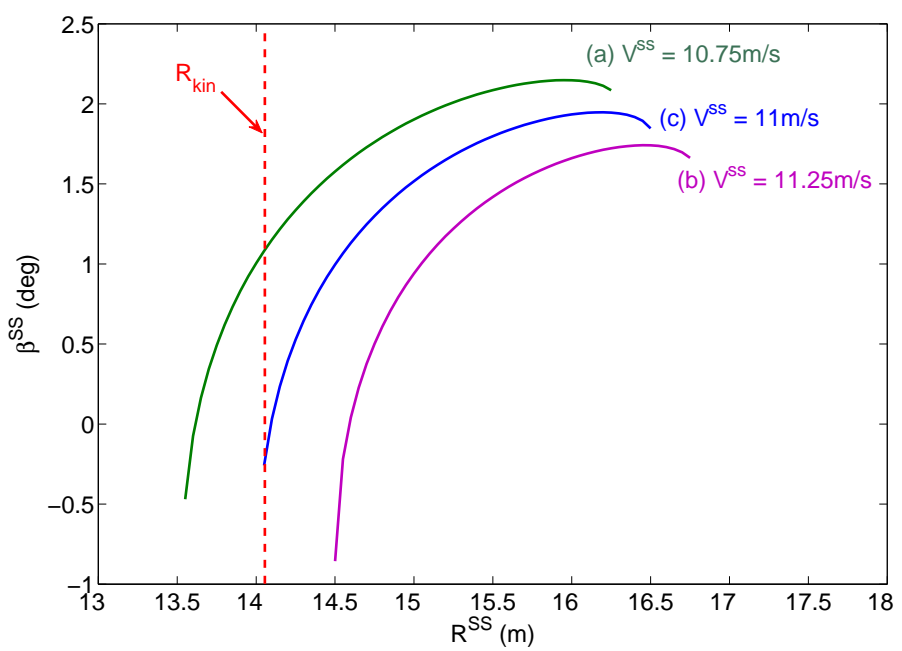

Figure 2. Selection of target steady-state according to the driver's steering angle command: (a) $R_{k i n}$ feasible at $V^{\mathrm{ss}}=10.75 \mathrm{~m} / \mathrm{s}$; (b) $R_{k i n}$ not feasible at $V^{\mathrm{ss}}=11.25 \mathrm{~m} / \mathrm{s}$; (c) $R_{k i n}$ coincides with the minimum calculated $R^{\mathrm{ss}}$ at $V^{\mathrm{ss}}=11 \mathrm{~m} / \mathrm{s}$.

\section{MPC Formulation}

We now design the sampled-data MPC strategy by discretizing the continuous-time plant and the associated quadratic cost function. For the nonlinear continuous-time system with state vector $x$, input vector $u$ and output vector $y$

$$
\dot{x}=f(x, u), \quad y=g(x, u),
$$

linearized about the equilibrium point $\left(x^{\mathrm{ss}}, u^{\mathrm{ss}}, y^{\mathrm{ss}}\right)$

$$
\dot{\tilde{x}}=A_{c} \widetilde{x}+B_{c} \widetilde{u}, \quad \widetilde{y}=C_{c} \widetilde{x}+D_{c} \widetilde{u},
$$

with associated cost function state and input weighting matrices $Q_{c}$ and $L_{c}$ respectively and cross-weighting matrix $M_{c}$, the discrete-time model using an exact discretization [2] and sampling time $T_{s}$ is

$$
\widetilde{x}_{k+1}=A_{d} \widetilde{x}_{k}+B_{d} \widetilde{u}_{k}, \quad \widetilde{y}_{k}=C_{d} \widetilde{x}_{k}+D_{d} \widetilde{u}_{k} .
$$

In the above equations, $\widetilde{x}=x-x^{\mathrm{ss}}, \widetilde{u}=u-u^{\mathrm{ss}}$ and $\widetilde{y}=y-y^{\mathrm{ss}}$ are the state, input and output errors from the equilibrium point, $A_{c}, B_{c}, C_{c}$ and $D_{c}$ are the Jacobian matrices 
from linearization and $A_{d}, B_{d}, C_{d}$ and $D_{d}$ are the discretized equivalents.

Then, assuming $D_{d}=D_{c}=0$ (no feedthrough term) and $C_{d}=I^{n}$ (full state feedback), the MPC regulation problem with horizon $N=N_{p}=N_{u}$ is

$$
\begin{aligned}
\operatorname{minimize} & \widetilde{x}_{N}^{T} P \widetilde{x}_{N}+\sum_{i=0}^{N-1}\left(\widetilde{x}_{i}^{T} Q \widetilde{x}_{i}+\widetilde{u}_{i}^{T} L \widetilde{u}_{i}+2 \widetilde{x}_{i}^{T} M \widetilde{u}_{i}\right), \\
\text { subject to } & \widetilde{x}_{0}=\widetilde{x}_{c u r}, \\
& \widetilde{x}_{i+1}=A_{d} \widetilde{x}_{i}+B_{d} \widetilde{u}_{i}, \quad i=0,1, \ldots, N-1 \\
& \widetilde{u}_{i}^{\text {min }} \leq \widetilde{u}_{i} \leq \widetilde{u}_{i}^{\text {max }}, \quad i=0,1, \ldots, N-1 \\
& \widetilde{x}_{i}^{\text {min }} \leq \widetilde{x}_{i} \leq \widetilde{x}_{i}^{\text {max }}, \quad i=1,2, \ldots, N
\end{aligned}
$$

where (2a) is the cost function [2], (2b) sets the initial state error $\widetilde{x}_{0}$ equal to the current one, $(2 \mathrm{c})$ are the discrete system dynamics and $(2 \mathrm{~d})-(2 \mathrm{e})$ are the state and input inequality constraints. The positive (semi-)definite matrix $Q$ and positive definite matrix $L$ are the weighting matrices on the state error and control effort respectively, and the positive definite matrix $M$ is the cross-weighting matrix. The inclusion of the terminal penalty $\widetilde{x}_{N}^{T} P \widetilde{x}_{N}$ in (2a) ensures closed-loop stability [18], with the matrix $P$ selected as the solution of the Discrete Algebraic Riccati Equation

$$
P=A_{c}^{T} P A_{c}+Q\left(B_{c}^{T} P A_{c}+M^{T}\right)^{T}\left(L+B_{c}^{T} P B_{c}\right)^{-1}\left(B_{c}^{T} P A_{c}+M^{T}\right) .
$$

Based on the standard MPC problem (2) a dense MPC formulation using soft constraints on the state [18] is used in this paper, with the necessary $A_{c}, B_{c}, C_{c}$ and $D_{c}$ matrices updated at each time step according to the current steering command from the driver and the current vehicle velocity using the analysis of section 2.2 . The resulting Quadratic Program (QP) problem is then solved using the active-set method [20] available through the quadprog command in MATLAB.

The main disadvantage of using an MPC strategy is its computational burden, which is directly related to the time that is needed to construct and solve the MPC problem. Many factors have a decisive role in this: the number of optimization variables and the number of constraints, along with the selected sampling time and horizon, can result in a large optimization problem that is too difficult to solve in real time. Based on these observations, in this following sections we present two MPC strategies using internal models of different complexity. In the first MPC strategy, called hereafter 'MPCt' (where "t" stands for "torque" input), we use the full four-wheel model (1) hence both the vehicle dynamics and the much faster wheel speed dynamics are included in the internal model, and the input is set as the two torques on the rear wheels. In the second MPC strategy, called hereafter 'MPCs' (where "s" stands for "slip" input), we use a smaller internal model which neglects the wheel speed dynamics, while the input is set as the longitudinal slips at the rear wheels. Then a Sliding Mode Slip Controller is used to calculate the necessary torques on the rear wheels according to the requested longitudinal slips.

\subsection{MPCt: MPC using Wheel Torque Inputs}

For the MPCt the full four-wheel model (1) is used, hence (neglecting the free-rolling front wheels) we set $x=\left[V \beta \dot{\psi} \omega_{R L} \omega_{R R}\right]^{T}$, and $u=\left[T_{R L} T_{R R}\right]^{T}$ (Fig. 3).

When defining the optimal control problem, the cross-weighting matrix $M_{c}$ is set to 


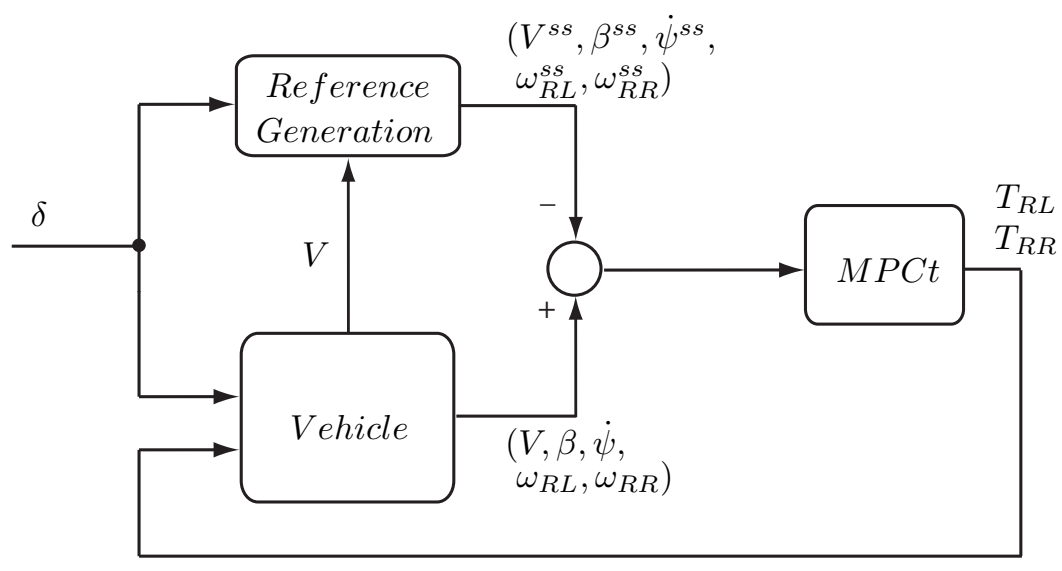

Figure 3. MPCt control structure.

zero (note that after discretization of the system, $M$ in (2a) will usually not be zero), while the weighting matrices $Q_{c}$ and $L_{c}$ are updated at each time step according to

$$
\begin{gathered}
Q_{c}=\operatorname{diag}\left\{q_{V}\left(\frac{1}{V_{\max }}\right)^{2},\left(\frac{1}{\beta_{\max }}\right)^{2},\left(\frac{1}{\dot{\psi}_{\max }}\right)^{2},\left(\frac{1}{\omega_{R L_{\max }}}\right)^{2},\left(\frac{1}{\omega_{R R_{\max }}}\right)^{2}\right\}, \\
L_{c}=\operatorname{diag}\left\{\left(\frac{1}{T_{R L_{\max }}}\right)^{2},\left(\frac{1}{T_{R R_{\max }}}\right)^{2}\right\},
\end{gathered}
$$

where the maximum values are set according to the steering input from the driver and the steady-state cornering analysis of section (2.2), along with the electric motor characteristics. In this way, the $Q_{c}$ and $L_{c}$ matrices are normalized with respect to the state and input vectors, and the only tuning parameter is $q_{V}$.

\subsubsection{State Constraints}

In order to avoid large yaw rate values, a yaw rate constraint according to the current velocity $V_{\text {cur }}$ is imposed at the beginning of the optimization and fixed throughout the prediction horizon. This constraint is based on the lateral acceleration limit for the current velocity and is coupled to the tyre/road friction coefficient $\mu_{\max }[21]$ :

$$
|\dot{\psi}| \leq \mu_{\max } g / V_{\text {cur }}
$$

Following $[4,14]$, a constraint on the maximum sideslip angle is also set according to the current velocity:

$$
|\beta|=\left\{\begin{array}{lll}
2 \frac{k_{1}-k_{2}}{V_{c h}^{3}} V_{c u r}^{3}-3 \frac{k_{1}-k_{2}}{V_{c h}^{2}} V_{c u r}^{2}+k_{1}, & \text { if } & V_{c u r}<V_{c h} \\
k_{2}, & \text { if } & V_{c u r} \geq V_{c h}
\end{array},\right.
$$

where $V_{c h}$ is the characteristic velocity of the vehicle defined under steady-state cornering conditions as the velocity at which a steering angle double the Ackerman angle is required for the vehicle to maintain the same turning radius [9]. The positive constants $k_{1}$ and $k_{2}$ are tuning parameters, chosen at $10 \pi / 180$ and $3 \pi / 180$ respectively. No constraints on the velocity or the rear wheel speeds are imposed. 


\subsubsection{Input Constraints}

For the MPCt the use of the two torques on the rear wheels as input of the internal model gives us the opportunity to set constraints on them based on the static torque map of the motor used.

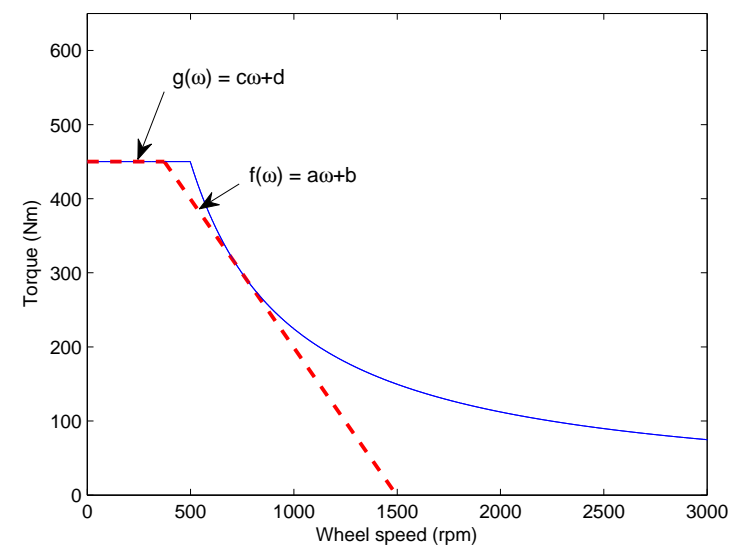

Figure 4. The static torque map and its approximation using affine functions of $\omega$.

The maximum torque achievable on each of the two rear wheels of the car is a nonlinear function of the corresponding wheel speed. Since the MPC problem (2) is formulated as a convex optimization problem that allows only for affine inequality constraints, the static torque map is approximated by two affine functions of $\omega$ as seen in Fig. 4. Taking for example the line $f=a \omega+b$ in Fig. 4 , we want

$$
T_{i j} \leq a \omega_{i j}+b
$$

and since for the MPC problem the system is linearized about the equilibrium point $\left(x^{\mathrm{ss}}, u^{\mathrm{ss}}\right)$, for $T=T^{\mathrm{ss}}+\widetilde{T}$ and $\omega=\omega^{\mathrm{ss}}+\widetilde{\omega}$ the above inequality gives

$$
T_{i j}^{\mathrm{ss}}+\widetilde{T}_{i j} \leq a\left(\omega_{i j}^{\mathrm{ss}}+\widetilde{\omega}_{i j}\right)+b \Rightarrow \widetilde{T}_{i j}-a \widetilde{\omega}_{i j} \leq a \omega_{i j}^{\mathrm{ss}}+b-T_{i j}^{\mathrm{ss}}
$$

Expanding then to both the rear wheels' torques with $V=V^{\mathrm{ss}}+\widetilde{V}, \beta=\beta^{\mathrm{ss}}+\widetilde{\beta}$ and $\dot{\psi}=\dot{\psi}^{\text {ss }}+\widetilde{\dot{\psi}}$ we get

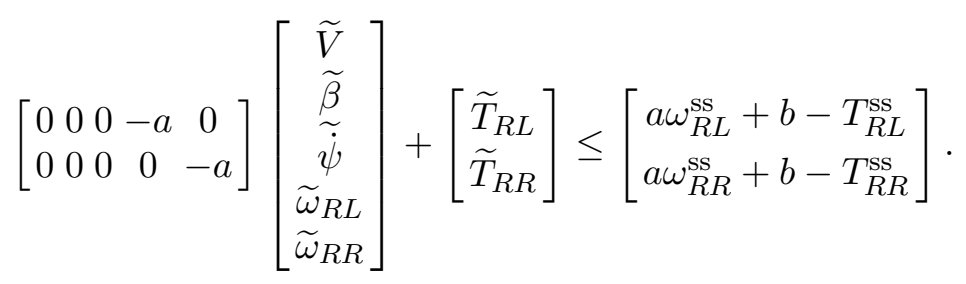

A similar procedure can be followed for line $g=c \omega+d$. The resulting polyhedron, as seen in Fig. 4, is convex and can be therefore used to define the input inequality constraints in (2).

In this paper, the above piecewise linear approximation of the static torque map is chosen in such way so that the error from the nonlinear constraint is minimized at a 
realistic range of wheel speeds. To this end, we choose $c$ and $d$ so that $g(\omega)$ coincides with the constant torque line (note that in this case $c=0$ ), and $a$ and $b$ so that $f(\omega)$ is tangent to the constant power curve and meets the longitudinal line at a high wheel speed.

\subsubsection{Longitudinal Slip Constraints}

Since, for stability reasons, it is deemed necessary to constrain the longitudinal slips on the rear wheels, another constraint on the state is also constructed for the MPCt to address this. Through the longitudinal slip definition

$$
s_{i j x}=\frac{V_{i j x}-\omega_{i j} r}{\omega_{i j} r}
$$

we can impose a constraint on the relative wheel speed

$$
s_{i j x}^{l} \leq s_{i j x} \leq s_{i j x}^{h} \Rightarrow\left\{\begin{array} { c } 
{ s _ { i j x } \leq s _ { i j x } ^ { h } } \\
{ - s _ { i j x } \leq - s _ { i j x } ^ { l } }
\end{array} \Rightarrow \left\{\begin{array}{c}
\frac{V_{i j x}-\omega_{i j} r}{\omega_{i j} r} \leq s_{i j x}^{h} \\
-\frac{V_{i j x}-\omega_{i j} r}{\omega_{i j} r} \leq-s_{i j x}^{l}
\end{array} .\right.\right.
$$

Then, linearizing the longitudinal tyre velocity about the equilibrium point, the above double inequality (7) can be expressed in terms of the state $\left[\begin{array}{ll}V & \beta\end{array}\right]^{T}$ to be used in the optimization problem. Taking for example the upper bound, we have

$$
\left(V_{i j x}^{\mathrm{ss}}+\frac{\vartheta V_{i j x}}{\vartheta V} \widetilde{V}+\frac{\vartheta V_{i j x}}{\vartheta \beta} \widetilde{\beta}+\frac{\vartheta V_{i j x}}{\vartheta \dot{\psi}} \widetilde{\dot{\psi}}\right)-\left(1+s_{i j x}^{h}\right) r\left(\omega_{i j}^{\mathrm{ss}}+\widetilde{\omega}_{i j}\right) \leq 0,
$$

and expanding to both the rear wheels, we get

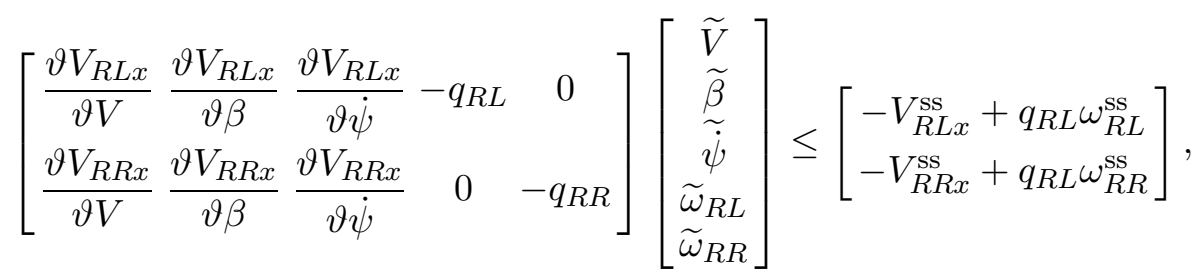

where

$$
q_{R L}=\left(1+s_{R L x}^{h}\right) r, \quad q_{R R}=\left(1+s_{R R x}^{h}\right) r .
$$

\subsection{MPCs: MPC Neglecting the Wheel Speed Dynamics}

The second MPC strategy neglects the fast wheel speed dynamics, so that a simpler internal model is used with $x=\left[\begin{array}{lll}V & \beta & \psi\end{array}\right]^{T}$ and $u=\left[\begin{array}{ll}s_{R L x} & s_{R R x}\end{array}\right]^{T}$. Then a Sliding Mode Slip Controller computes the necessary torques on the rear wheels based on the requested longitudinal slips (Fig. 5). When defining the optimal control problem, the cross-weighting matrix $M_{c}$ is set again to zero, while the weighting matrices $Q_{c}$ and $L_{c}$ are set in a way similar to the MPCt strategy. 


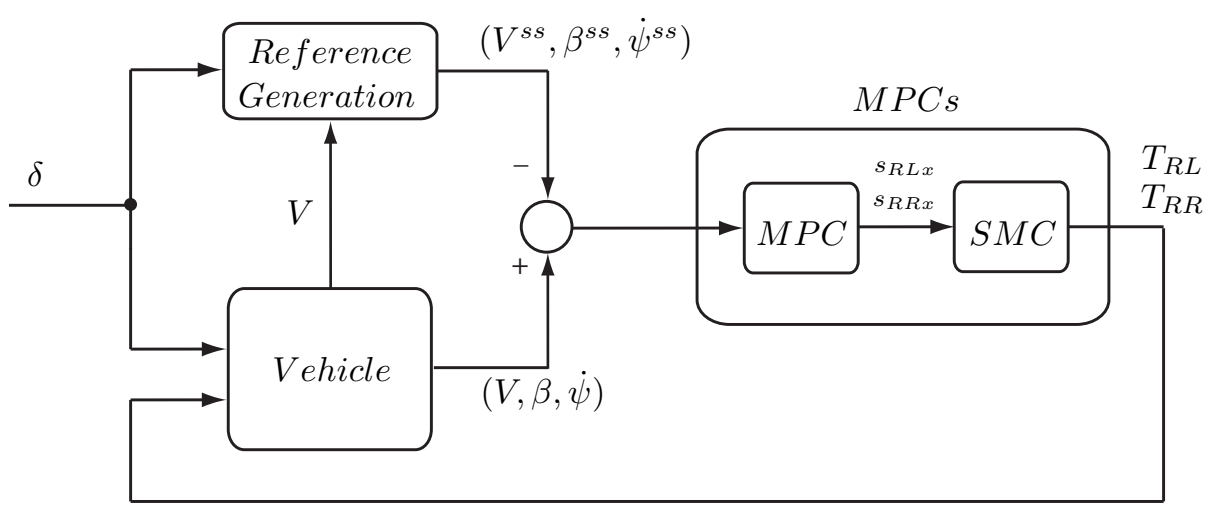

Figure 5. MPCs control structure.

\subsubsection{State, Input and Torque Constraints}

For the MPCs we use the same yaw rate and sideslip angle constraints (4)-(5) as with the MPCt, while no constraint is set on the vehicle velocity.

Constraints are also set for the input $u=\left[\begin{array}{ll}s_{R L x} & s_{R R x}\end{array}\right]^{T}$ so that the longitudinal slips on the rear wheels never exceed the maximum allowable slip for safe operation of the vehicle. Using the tyre parameters as found in Table 1 in the simplified MF [3] we set the constraint

$$
\left|s_{R j x}\right| \leq 0.07
$$

For the MPCs we can not directly account for the motor limits in the form of its static torque map as was the case with the MPCt. We therefore construct an additional constraint on the state and input in order to avoid excessive torque requests from the two motors. Neglecting the wheel speed dynamics, the longitudinal tyre force on each of the rear wheels can be bounded by the maximum and minimum allowable torques

$$
T_{i j}^{l} \leq f_{i j x} r \leq T_{i j}^{h}
$$

where $T_{i j}^{l}$ and $T_{i j}^{h}$ are calculated as a function of the current wheel speed according to the static torque map at the beginning of each time step and fixed for the rest of the prediction horizon. Then, linearizing the longitudinal tyre force about the equilibrium point, the above double inequality (9) can be expressed in terms of the state $\left[\begin{array}{ll}V & \beta\end{array}\right]^{T}$ and input $\left[\begin{array}{ll}s_{R L x} & s_{R R x}\end{array}\right]^{T}$ to be used in the optimization problem. Taking for example the upper bound

$$
f_{i j x}^{\mathrm{ss}}+\frac{\vartheta f_{i j x}}{\vartheta s_{i j x}} \widetilde{s}_{i j x}+\frac{\vartheta f_{i j x}}{\vartheta V} \widetilde{V}+\frac{\vartheta f_{i j x}}{\vartheta \beta} \widetilde{\beta}+\frac{\vartheta f_{i j x}}{\vartheta \dot{\psi}} \widetilde{\dot{\psi}} \leq \frac{T_{i j}^{h}}{r}
$$

and expanding to both rear wheels

$$
\left[\begin{array}{ccc}
\frac{\vartheta f_{R L x}}{\vartheta V} & \frac{\vartheta f_{R L x}}{\vartheta \beta} & \frac{\vartheta f_{R L x}}{\vartheta \dot{\psi}} \\
\frac{\vartheta f_{R R x}}{\vartheta V} & \frac{\vartheta f_{R R x}}{\vartheta \beta} & \frac{\vartheta f_{R R x}}{\vartheta \dot{\psi}}
\end{array}\right]\left[\begin{array}{c}
\widetilde{V} \\
\widetilde{\beta} \\
\widetilde{\psi}
\end{array}\right]+\left[\begin{array}{ll}
\frac{\vartheta f_{R L x}}{\vartheta s_{R L x}} & \frac{\vartheta f_{R L x}}{\vartheta s_{R R x}} \\
\frac{\vartheta f_{R R x}}{\vartheta s_{R L x}} & \frac{\vartheta f_{R R x}}{\vartheta s_{R R x}}
\end{array}\right]\left[\begin{array}{c}
\widetilde{s}_{R L x} \\
\widetilde{s}_{R R x}
\end{array}\right] \leq\left[\begin{array}{l}
\frac{T_{R L}^{h}}{r}-f_{R L x}^{\mathrm{ss}} \\
\frac{T_{R R}^{h}}{r}-f_{R R x}^{\mathrm{ss}}
\end{array}\right] .
$$




\subsubsection{Sliding Mode Slip Controller}

The torque demand on the two electric motors according to the longitudinal slip requests are then calculated using a Sliding Mode Slip Controller, constructed in a way similar to $[11,25]$.

From the longitudinal slip definition (6) the slip dynamics are

$$
\dot{s}_{i j x}=\frac{d}{d t}\left(\frac{V_{i j x}-\omega_{i j} r}{\omega_{i j} r}\right)=\frac{\dot{V}_{i j x} \omega_{i j} r-V_{i j x} \dot{\omega}_{i j} r}{\omega_{i j}^{2} r^{2}}=\frac{\dot{V}_{i j x}}{\omega_{i j} r}-\frac{V_{i j x} \dot{\omega}_{i j}}{\omega_{i j}^{2} r},
$$

and using (6) and (1d),

$$
\dot{s}_{i j x}=\left(s_{i j x}+1\right) \frac{\dot{V}_{i j x}}{V_{i j x}}-\left(s_{i j x}+1\right)^{2} \frac{r}{I_{w} V_{i j x}}\left(T_{i j}-f_{i j x} r\right) .
$$

Setting the sliding surface as $e=s_{i j x}-s_{i j x}^{\text {des }}$ and using $L=(1 / 2) e^{2}$ as a Lyapunov function candidate with $\dot{e}=\dot{s}_{i j x}$ (assuming that $s_{i j x}^{\text {des }}$ remains constant), we have [13]

$$
\begin{aligned}
\dot{L} & =e \dot{e}=e \dot{s}_{i j x}=e\left(\left(s_{i j x}+1\right) \frac{\dot{V}_{i j x}}{V_{i j x}}-\left(s_{i j x}+1\right)^{2} \frac{r}{I_{w} V_{i j x}}\left(T_{i j}-f_{i j x} r\right)\right) \\
& \left.=e\left(\left(s_{i j x}+1\right) \frac{\dot{V}_{i j x}}{V_{i j x}}-\left(s_{i j x}+1\right)^{2} \frac{r}{I_{w} V_{i j x}} T_{i j}+\left(s_{i j x}+1\right)^{2} \frac{r}{I_{w} V_{i j x}} f_{i j x} r\right)\right),
\end{aligned}
$$

and taking

$$
\begin{aligned}
T_{i j} & =-\frac{I_{w} V_{i j x}}{\left(s_{i j x}+1\right)^{2} r}\left(-\left(s_{i j x}+1\right) \frac{\dot{V}_{i j x}}{V_{i j x}}-\left(s_{i j x}+1\right)^{2} \frac{R}{I_{w} V_{i j x}} f_{i j x} r-\eta \operatorname{sgn}(e)\right) \\
& =\frac{I_{w} \dot{V}_{i j x}}{\left(s_{i j x}+1\right) r}+r f_{i j x}+\frac{I_{w} V_{i j x}}{\left(s_{i j x}+1\right)^{2} R} \eta \operatorname{sgn}(e),
\end{aligned}
$$

with $\eta$ a small positive number yields

$$
\dot{L}=-e \eta \operatorname{sgn}(e)=-\eta|e|<0 \text {. }
$$

Then defining $\kappa=\frac{I_{w} V_{i j x}}{\left(s_{i j x}+1\right)^{2} r} \eta$, the control law is

$$
T_{i j}=\frac{I_{w} \dot{V}_{i j x}}{\left(s_{i j x}+1\right)^{2} r}+r f_{i j x}+\kappa \operatorname{sgn}(e)
$$

or to reduce chattering we can use instead

$$
T_{i j}=\frac{I_{w} \dot{V}_{i j x}}{\left(s_{i j x}+1\right)^{2} r}+r f_{i j x}+\kappa \mathrm{sat}\left(\frac{e}{\Delta}\right),
$$


where $\operatorname{sat}(\cdot)$ the saturation function

$$
\operatorname{sat}(y)= \begin{cases}y, & \text { if }|y| \leq 1 \\ \operatorname{sgn}(y), & \text { if }|y|>1\end{cases}
$$

and $\Delta$ a positive constant [13].

Remark: While some of the signals needed for both the MPCt and MPCs strategies can be measured using standard equipment in modern vehicles (yaw rate, wheel speeds, steering angle), others need to be estimated (velocity, sideslip angle, tyre slips and torques).This is indeed possible, as shown in [1,23] and is part of the on-going work before implementing the controller on a prototype vehicle.

\section{Tuning of the two MPC Strategies}

After choosing the internal model for the MPC problem (2), two are the most important parameters affecting both the performance and computational burden for an MPC formulation: the sampling time $T_{s}$ and the horizon $N$. For the evaluation of the performance of the two different MPC strategies we use the closed-loop cost, defined as the summation of the running cost

$$
J_{c l}=\sum_{k=0}^{\left\lceil\frac{T_{s i m}-T_{s}}{T_{s}}\right\rceil}\left(x_{k}^{T} Q x_{k}+u_{k}^{T} L u_{k}+2 x_{k}^{T} M u_{k}\right),
$$

where $T_{\text {sim }}$ is the chosen simulation time and $\lceil\cdot\rceil$ is the ceiling function, which maps a real number to the smallest following integer. The above expression is the summation of the weighted square of the state error and the control effort for the duration of the simulation, hence the cost that the MPC tries to minimize at the first place, and can be therefore used as a metric of the controller's performance. For the evaluation of the computational effort, the mean time $T_{\text {comp }}$ required from the quadprog solver in MATLAB to construct and solve the MPC problem is used.

First we investigate the effect of varying the sampling time on the performance and the computational time of the two MPC strategies for a range of sampling times and a horizon equal to the simulation time of $T_{s i m}=10 \mathrm{~s}$. Next we check the effect of choosing a horizon that is smaller than the simulation time of $T_{\text {sim }}=10 \mathrm{~s}$. In this way, we will select the best sampling time and horizon combination for the two MPC strategies that provides the best compromise between performance and computational time. A set of simple simulation scenarios is used, whereas the vehicle is going straight and a step steer input is applied after $2 \mathrm{~s}$. It is assumed at this point that no acceleration or braking requests come from the driver. For each simulation, the initial vehicle velocity is chosen so that it is $1 \mathrm{~m} / \mathrm{s}$ higher from the maximum velocity allowable for the applied step steering input hence both MPC strategies will regulate the velocity, sideslip angle and yaw rate of the vehicle according to the analysis presented in section 2.2 .

\subsection{Impact of Varying the Sampling Time in the MPCt}

For the MPCt, using a sampling time above $0.035 \mathrm{~s}$ results in a controller that cannot be stabilizing anymore. This is the direct result of including the fast wheel speed dynamics 
in the internal model, and reveals the main disadvantage of this strategy: consideration of the wheel speed dynamics in the MPC problem increases the number of optimization variables while at the same time calls for faster sampling times.

Using the set of test scenarios described above, Fig. 6(a) shows the closed-loop cost for a range of step steering inputs and sampling times between 0.01-0.035s. No major variations are noticed for this range of sampling times. Looking more closely in the test results for a step steering input of 6deg, in Fig. 6(b) we observe that for sampling times below $0.02 \mathrm{~s}$, the time needed to construct and solve the QP problem increases exponentially. The pareto frontier in Fig. 6(c) shows a similar trend, with computational times increasing rapidly with only small gains in the closed-loop cost.

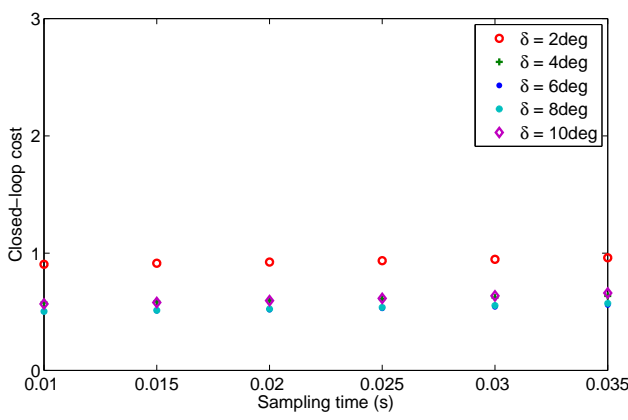

(a) $J_{c l}$ vs $T_{s}$ for different $\delta \mathrm{s}$
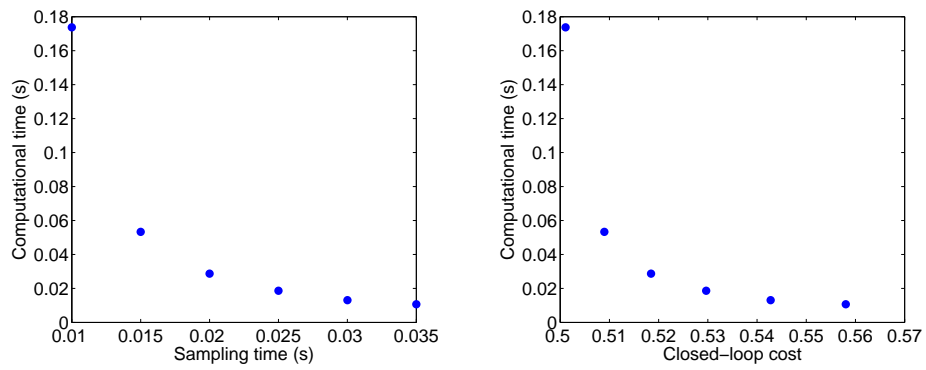

(b) $T_{\text {comp }}$ vs $T_{s}(\delta=6 \mathrm{deg})$

(c) $T_{\text {comp }}$ vs $J_{c l}(\delta=6 \mathrm{deg})$

Figure 6. Variation of closed-loop cost with sampling time $T_{s}=0.01-0.035 \mathrm{~s}$ is minimal for a range of step steering inputs $\delta$ (a), but computational time increases with lower sampling times (b) or costs (c) in the MPCt.

\subsection{Impact of Varying the Sampling Time in the MPCs}

Using the same set of test scenarios as in the case of the MPCt, Fig. 7(a) shows the variation of the closed-loop cost with sampling time for a range of step steer inputs for the MPCs. No considerable changes in performance for sampling times below $0.07 \mathrm{~s}$ can be noticed. Looking at the test results for a step steering input of 6deg, in Fig. 7(b) we observe that the computational time increases rapidly for sampling times below $0.05 \mathrm{~s}$, so there is a clear trade-off between closed-loop cost and computational time with changes in the sampling time, while a similar increase in computational time can be noticed for small reductions in the closed-loop cost below 1 (Fig. 7(c)). 


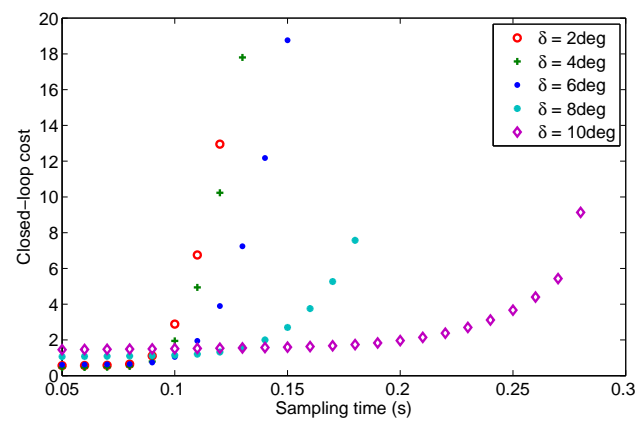

(a) $J_{c l}$ vs $T_{s}$ for different $\delta \mathrm{s}$
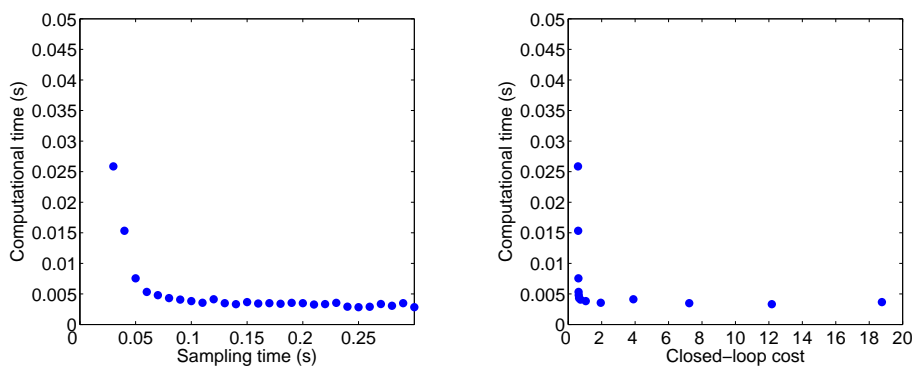

(b) $T_{\text {comp }}$ vs $T_{s}(\delta=6 \mathrm{deg})$

(c) $T_{\text {comp }}$ vs $J_{c l}(\delta=6 \mathrm{deg})$

Figure 7. Variation of closed-loop cost with sampling time is minimal for sampling times below $T_{s}=0.07 \mathrm{~s}$ across a range of step steering inputs $\delta(a)$, but computational time increases rapidly with sampling times lower than $0.05 \mathrm{~s}(\mathrm{~b})$, or costs lower than 1 (c) in the MPCs.

\subsection{Impact of Varying the Horizon in the MPCt and MPCs}

The horizon length of $10 \mathrm{~s}$ in combination with the short sampling times used in the above sections resulted in long computational times, a large portion of which was spend in constructing the matrices for the dense MPC problem. The increase in construction time with longer horizons in the MPCt and MPCs can be seen in Fig. 8. We also note that the relevant increase in construction time for the MPCs is lower to the one for the MPCt, a result of the smaller number of optimization variables used in the MPCs. However, in both strategies the construction time drops to values as low as $0.005 \mathrm{~s}$ for horizons less than 1 s.

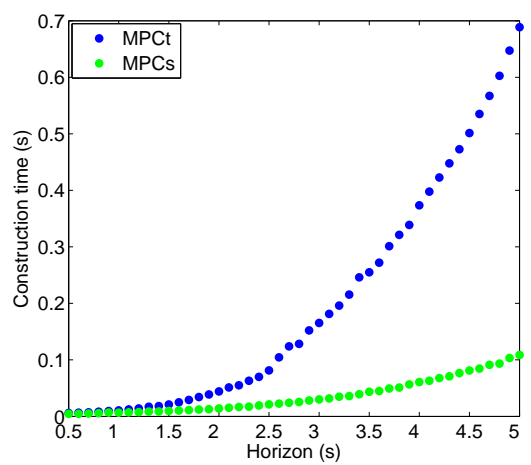

Figure 8. Construction time needed from the two MPC strategies with longer horizons. A larger grow rate is observed in the MPCt case, a direct result of the larger number of optimization variables used in this strategy when compared to the MPCs. 


\section{Comparison of the two MPC Strategies}

In the section to follow we compare the two MPC strategies against each other and against a LQR strategy [22] under different scenarios using the full four-wheel model (1) in Simulink environment. We also investigate the changes in tracking performance and computational time when a control horizon that is shorter than the prediction horizon is used. Based on the analysis presented in the previous section for the MPCt we set $T_{s}=0.035 \mathrm{~s}$ and $N=1 \mathrm{~s}$, and for the MPCs we set $T_{s}=0.05 \mathrm{~s}$ and $N=1 \mathrm{~s}$.

\subsection{Single Step Steering Input Scenario}

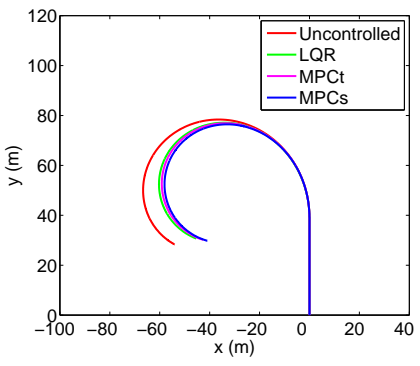

(a) Trajectory

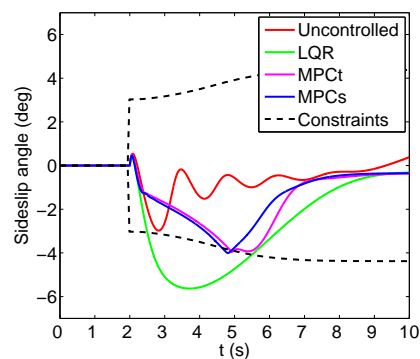

(c) Sideslip angle

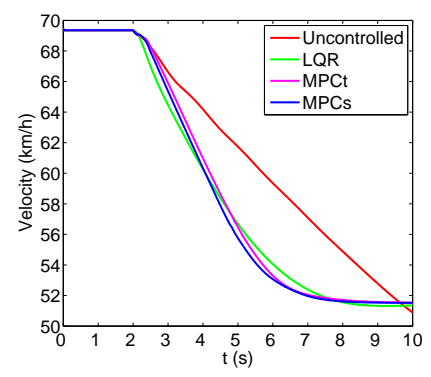

(b) Velocity

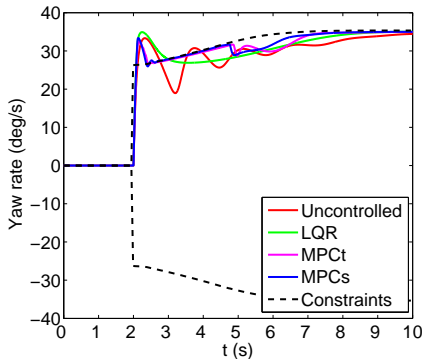

(d) Yaw rate

Figure 9. Simulation results for a step steering input of $\delta=6 \mathrm{deg}$ and an initial velocity which is $5 \mathrm{~m} / \mathrm{s}$ higher than the maximum possible. A similar tighter trajectory (a) and velocity regulation (b) for the MPCt, the MPCs and the LQR is observed, but at the expense of larger sideslip angle and yaw rate overshoots (c)-(d) for the LQR case.

The two MPC strategies are first evaluated under a single step steering input. The scenario under consideration is similar to the one used in section 4 but this time we set the initial velocity much higher, at $5 \mathrm{~m} / \mathrm{s}$ above the maximum possible steady-state velocity for the given step steering input. We assume again that no acceleration or braking commands come from the driver.

As we can see from Fig. 9(a), the uncontrolled vehicle traces a trajectory with a larger radius than the three controllers while exhibiting oscillations in both the sideslip angle and yaw rate responses (Fig. 9(c)-9(d)). Both the MPC strategies and the LQR show a tighter trajectory, but with some distinctive differences. While the velocity time histories for all three strategies look similar (Fig. 9(b)), the LQR exhibits a larger yaw rate overshoot (Fig. 9(d)) and a subsequent increase in sideslip angle (Fig. 9(c)). The two MPC strategies on the other hand restrict the yaw rate grow according to the yaw rate constraint (4) and achieve smaller overall sideslip angles along with reduced sideslip 
angle rates for the first couple of seconds after applying the step steering input. Note that, since the velocity time histories for the MPCt and MPCs are similar (Fig. 9(b)) and therefore the two velocity dependent yaw rate and sideslip angle constraints (4)-(5) propagate in time in a similar way for both the MPCt and the MPCs, for clarity reasons we choose to show in Fig 9(c)-9(d) only the constraints that correspond to the MPCt results.

\subsection{Double-Lane Change Scenario}

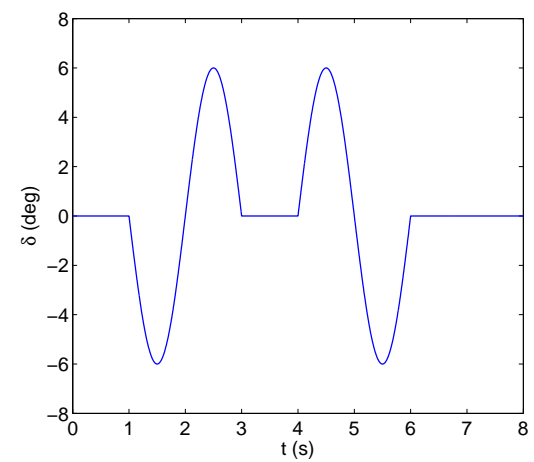

Figure 10. Open-loop steering input used in the double-lane change scenario.

As a second simulation scenario we use a fast double-lane change, whereas the steering input is set as a sinusoidal function of time (Fig. 10). This time the uncontrolled vehicle becomes unstable in the first part of the manoeuvre while the LQR shows an equally unstable behaviour (Fig. 11(a)). Both MPCt and MPCs keep the vehicle stable, with small yaw rate overshoots (Fig. 11(d)) and sideslip angle values well within their maximum values (Fig. 11(c)). Note that the sideslip angle and yaw rate constraints shown correspond again to the MPCt results.

Looking at the longitudinal slip time histories on the rear wheels in Fig. 11(e)-11(f), we see that the two MPC strategies keep the slip within the bound (8). It is interesting to note that the longitudinal slips on the rear wheels for the LQR case hit saturation levels only for the second part of the manoeuvre where the vehicle is already unstable, hence the source of instability in this scenario is the large yaw rate and sideslip angle values. The high frequency oscillations noticed for the LQR are the result of the switching strategy used in this case to keep the controller active only within the maximum longitudinal slip threshold [22].

\subsection{Impact of Varying the Control Horizon on the MPC Performance and Computational Time}

Up until now the control horizon in MPCt and MPCs was set equal to the prediction horizon, $N_{u}=N_{p}=1 \mathrm{~s}$. In this section we examine the effect of reducing the control horizon while keeping the prediction horizon fixed at $N_{p}=1$ s so that the computational time is always below the sampling time. For this, we choose a very aggressive double step steering input scenario, whereas the vehicle is going straight and a step steering of -6deg is applied at $2 \mathrm{~s}$ followed by a step steering of $6 \mathrm{deg}$ at $3 \mathrm{~s}$, with an initial velocity of $3 \mathrm{~m} / \mathrm{s}$ higher than the maximum possible steady-state velocity. 


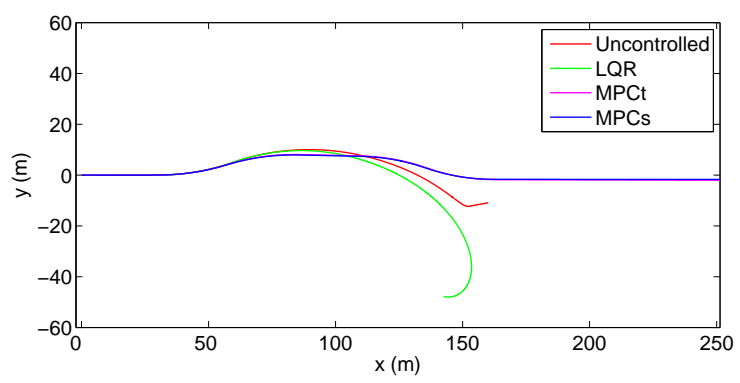

(a) Trajectory

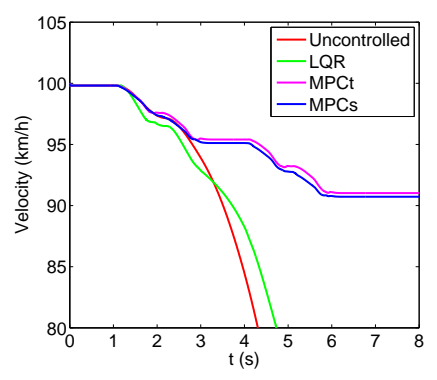

(b) Velocity

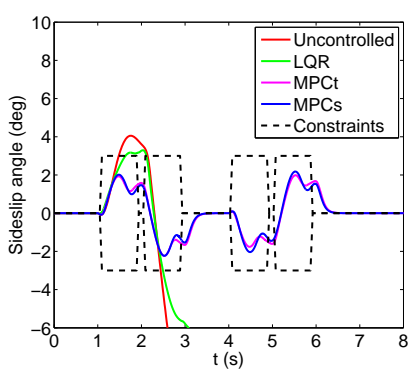

(c) Sideslip angle

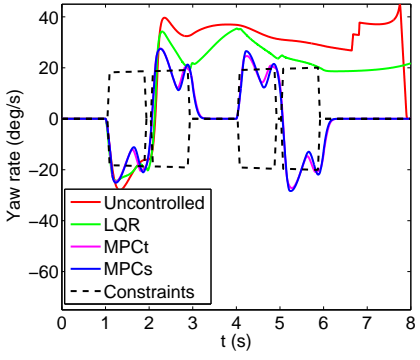

(d) Yaw rate

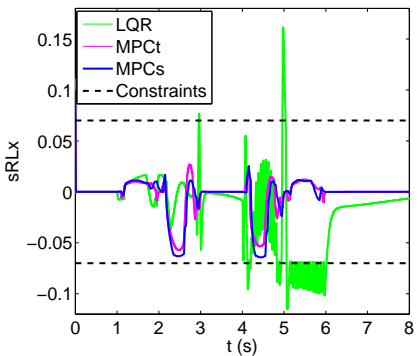

(e) Rear-left wheel long. slip

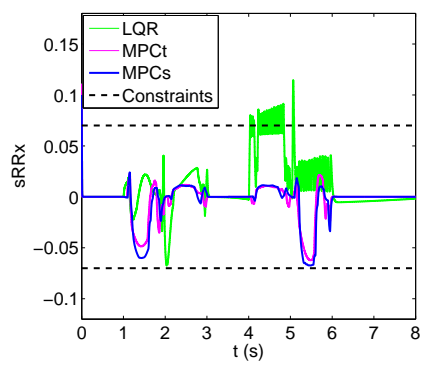

(f) Rear-right wheel long. slip

Figure 11. Both the MPCt and MPCs strategies successfully negotiate the manoeuvre with small sideslip angle and yaw rate values (c)-(d). The LQR on the other hand can not keep the vehicle stable due to large sideslip angle and yaw rate values (c)-(d) despite the switching strategy used in this case to keep the controller active only within the maximum longitudinal slip threshold (e)-(f).

\subsubsection{Impact of Varying the Control Horizon in the MPCt}

As we can see from Fig. 12, in order to drop the computational time at levels below the sampling time of $T_{s}=0.035 \mathrm{~s}$ the control horizon needs to be reduced to $N_{u}=0.28 \mathrm{~s}$ (Fig. 12(b)). From Fig. 12(c) we observe that using the shorter horizon results in a slower drop in velocity after the first step steering input but a faster drop after the second step steering input (constraints shown only for the shorter horizon case), a result of the higher sideslip angle values experienced for this part of the manoeuvre (Fig. 12(d)). We can conclude that using the shorter control horizon has a noticeable performance degradation in the controller performance in the case of the MPCt.

\subsubsection{Impact of Varying the Control Horizon in the MPCs}

In the case of the MPCs, the relatively higher sampling time of $T_{s}=0.05 \mathrm{~s}$ allows for a longer control horizon and a smaller loss in performance. Fig. 13(a)-13(b) show that reducing the control horizon to $N_{u}=0.5 \mathrm{~s}$ reduces the computational time without af- 


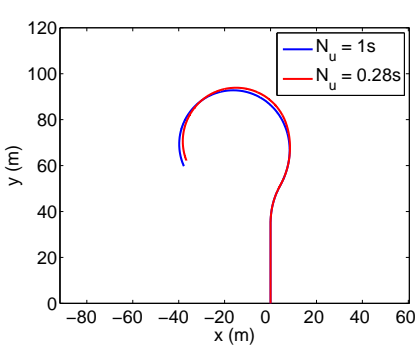

(a) Trajectory

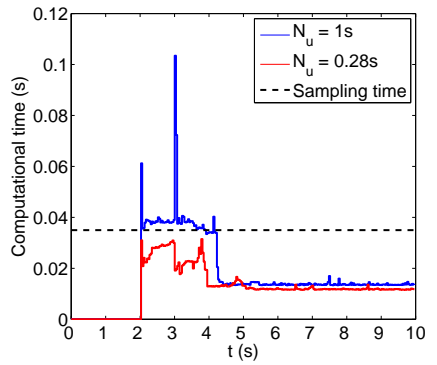

(b) Computational time

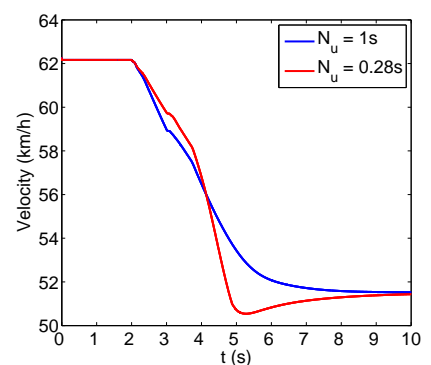

(c) Velocity

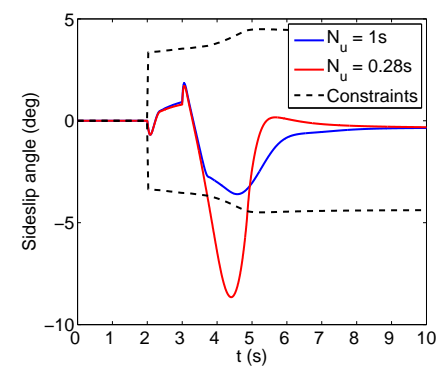

(d) Sideslip angle

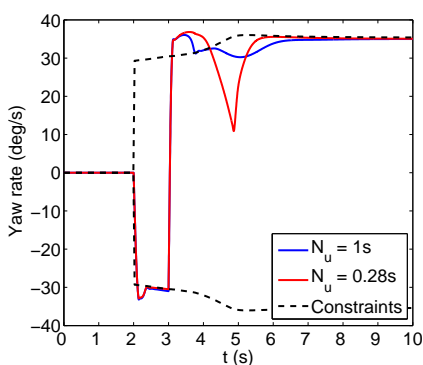

(e) Yaw rate

Figure 12. Use of a shorter control horizon of $N_{u}=0.28 \mathrm{~s}$ in the MPCt in order to keep the computational time below the sampling time (b) leads to performance degradation (c)-(e).

fecting the vehicle trajectory. From Fig. 13(b) it is also interesting to note that, apart from the two spikes at $2 \mathrm{~s}$ and $3 \mathrm{~s}$, the computational time is already lower than the sampling time even without the shorter control horizon. The smaller impact in performance in the case of the MPCs can also be confirmed by the velocity, sideslip angle and yaw rate time histories (Fig. 13(c)-13(e)) which show no major differences when the shorter control horizon is used (constraints shown only for the shorter horizon case).

\section{Parameter Sensitivity Analysis}

Using a simple step steering input scenario in Simulink, in this section we check the robustness of the MPCs controller under variations of the vehicle's mass $m$ and mass moment of inertia $I_{z}$, along with variations in the parameter $D=\mu_{\max }$ of the MF which represents the maximum value of the friction coefficient for a given road condition. In the driving scenario considered here, the vehicle is initially going straight with a speed of $14 \mathrm{~m} / \mathrm{s}$ and a step steer input of $10 \mathrm{deg}$ is applied after $2 \mathrm{~s}$ (note that the initial velocity is $5 \mathrm{~m} / \mathrm{s}$ higher than the maximum velocity allowable for this applied step steering input). For this test we set $\mu_{\max }=0.6$ which corresponds to wet road conditions, while both $m$ and $I_{z}$ are kept to their nominal values according to Table 1.

As we can see from Fig.14(a)-14(c), variations in the vehicle's mass results in small steady state errors, while also causes sideslip angle and yaw rate oscillations in the case of the heavier vehicle. From Fig.14(d)-14(f) we can see that variations in the vehicle's mass moment of inertia seem to have minimal impact on the response. The same cannot be said however for variations in the tyre/road friction coefficient, as seen in Fig.14(g)-14(i). While small steady state errors are again observed, a drop of 0.2 in the value of $\mu_{\max }$ results also in rather large oscillations in the yaw rate response.

We can conclude that the MPCs controller is robust against variations in $m, I_{z}$ and 


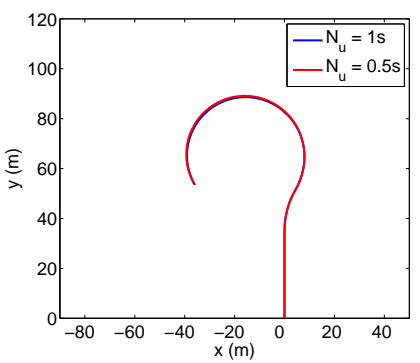

(a) Trajectory

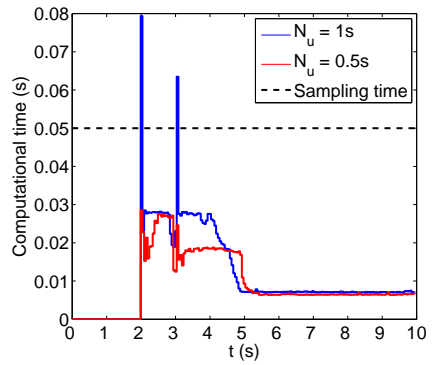

(b) Computational time

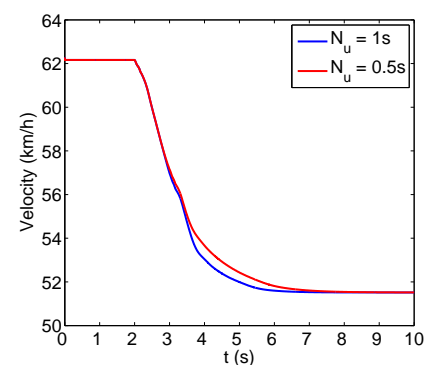

(c) Velocity

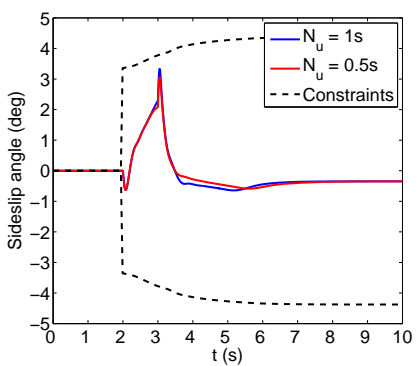

(d) Sideslip angle

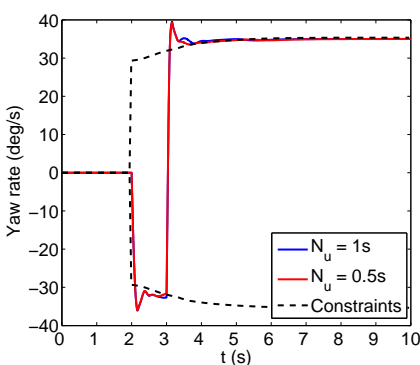

(e) Yaw rate

Figure 13. No observable performance degradation can be noticed (c)-(e) when a shorter control horizon of $N_{u}=0.5 \mathrm{~s}$ is used in the MPCs in order to keep the computational time below the sampling time (b).

$\mu_{\max }$ although the importance of good measurement and/or estimation is emphasised, especially in the case of estimating the road conditions.

\section{Validation with a High Fidelity Vehicle Model}

In the following section we compare the more efficient MPCs strategy against the LQR $[22]$ and a vehicle without torque vectoring intervention in CarSim environment. The scenarios under consideration are similar to the ones presented in the previous section but with two important differences: 1) the use of the high fidelity vehicle model available in CarSim, and 2) the availability of a driver model in CarSim for defining the steering input in a closed-loop form.

Based on the results of sections $4-5$, for the MPCs we set $T_{s}=0.05 \mathrm{~s}, N_{p}=1 \mathrm{~s}$ and $N_{u}=0.5 \mathrm{~s}$. We assume again that no acceleration or braking commands come from the driver.

\subsection{U-turn Scenario}

For the U-turn scenario, we use the driver model in CarSim to steer the vehicle through a turn of $56 \mathrm{~m}$ radius. The road is $5.6 \mathrm{~m}$ wide and dry $(\mu=0.9)$, while the entry speed is $85 \mathrm{~km} / \mathrm{h}$.

In Fig. 15(a) we see the trajectory of the vehicle using the MPCs in blue, the vehicle using the LQR in green and the vehicle without torque vectoring intervention in red. Both the MPCs and the LQR keep the vehicle within the road limits while the uncontrolled vehicle exits the road. Apart from keeping the vehicle within the road limits, the early velocity drop from the MPC and LQR (Fig. 15(c)) allows also for smaller wheel steering inputs from the driver (Fig. 15(b)). In Fig. 15(d) we observe that, while the MPC keeps 


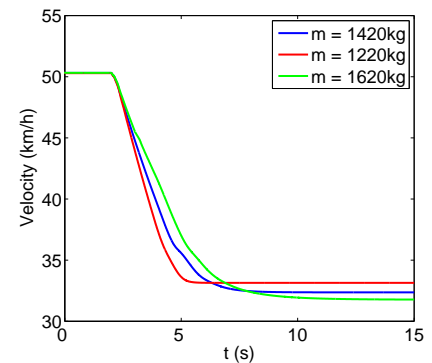

(a)

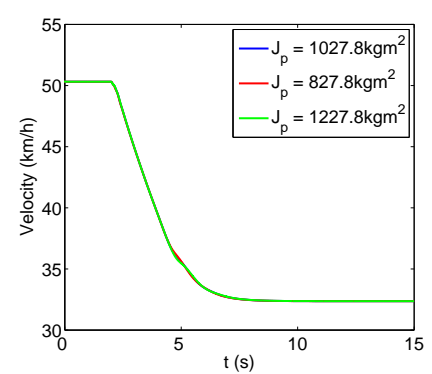

(d)

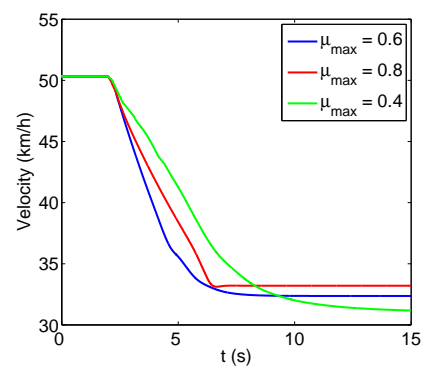

(g)

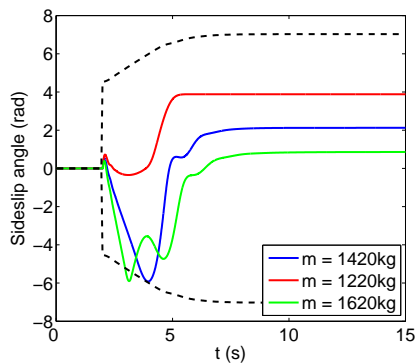

(b)

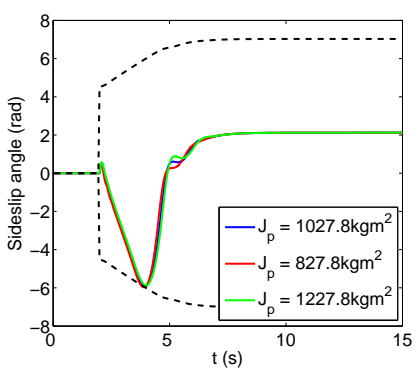

(e)

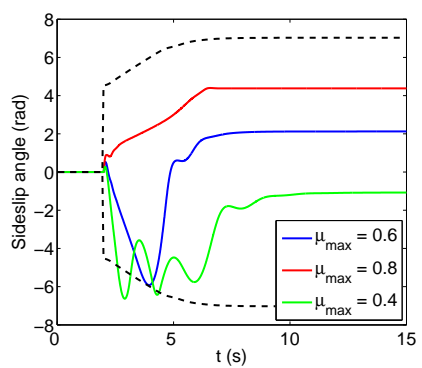

(h)

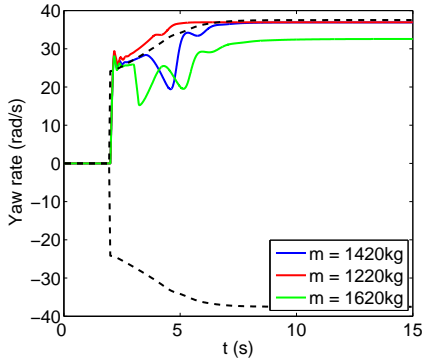

(c)

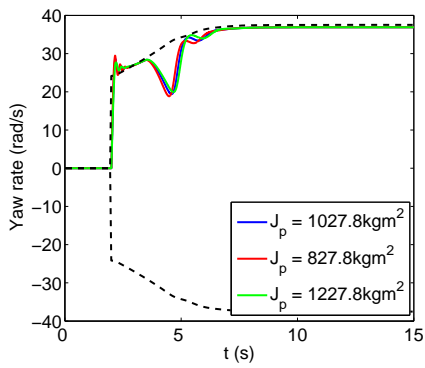

(f)

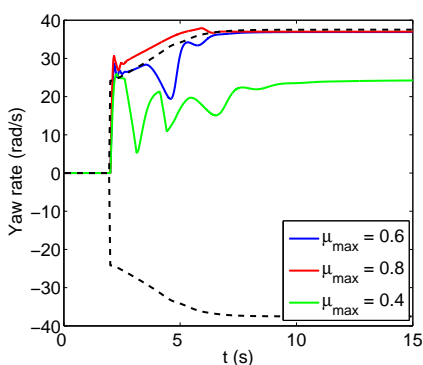

(i)

Figure 14. Vehicle's response remains largely unchanged with variations in the mass moment of inertia (d)-(f), but exhibits steady state errors and oscillations with variations in the vehicle's mass (a)-(c) and the tyre/road friction coefficient (g)-(i), however we can conclude that the controller is robust against variations in these parameters.

the sideslip angle values to levels comparable to the ones experienced by the uncontrolled vehicle, the vehicle using the LQR shows much larger values, a direct result of the inability of the LQR strategy to constrain the vehicle state.

From Fig. 15(f)-15(g) we see that for this scenario the longitudinal slip values for both the MPCs and the LQR stay in the linear region of the tyre. The small oscillations after $8 \mathrm{~s}$ for the MPCs case are related to the torque regulation from this strategy at this point. Finally, from Fig. 15(h)-15(i) we observe that the MPCs keeps for the most part the torque requests within the static torque map. Note here that, considering the power limitations of the electric motors, the actual torques applied on the rear wheels are always saturated according to the static torque map (Fig. 4).

From the U-turn scenario as analysed above, one could say that the MPCs gives a similar response to the simpler LQR. However the yaw rate regulation and most importantly the smaller sideslip angle values in the MPCs case show that the same performance can be achieved without having to compromise the stability of the vehicle, a point that becomes important in a fast manoeuvre as the double-lane change presented next. 


\subsection{Double-Lane Change Scenario}

For the double-lane change scenario we use again the driver model available in CarSim, but this time to follow a predefined path corresponding to a double-lane change manoeuver as denoted by a dashed line in Fig. 16(a). The road is assumed wet $(\mu=0.4)$, and the entry speed is set to $60 \mathrm{~km} / \mathrm{h}$.

Fig. 16(a) shows the trajectory of the vehicle using the MPCs in blue, the vehicle using the LQR in green and the vehicle without torque vectoring intervention in red. Both the uncontrolled vehicle and the vehicle with the LQR become unstable towards the end of the manoeuvre and spin out of control. While the wheel steering input (Fig. 16(b)) and the velocity time histories (Fig. 16(c)) for the MPCs and the LQR look similar for the first $5 \mathrm{~s}$, the large yaw rate and sideslip angle values in the case of the LQR result in an unstable condition from which the driver cannot recover. Finally, from Fig. 16(f)-16(i) we see that the MPCs keeps the longitudinal slips within the bounds (8). The high frequency oscillations noticed for the LQR are again the result of the switching strategy.

From the double-lane change scenario as analysed above, we see that the MPCs can stabilize the vehicle under a fast manoeuvre on a slippery road. The uncontrolled vehicle does not complete the test successfully while the use of the LQR results in an equally unstable behaviour due to the excessive yaw rate and sideslip angle values.

\section{Conclusions}

Two MPC strategies of different complexity for combined yaw, sideslip and velocity regulation have been presented. The first strategy, called MPCt, uses an internal model that includes both the vehicle dynamics and the much faster wheel speed dynamics as the state and the torque on the rear wheels as the input. The second strategy, called MPCs, neglects the wheel speed dynamics, hence uses only the vehicle dynamics as the state and the longitudinal slip on the rear wheels as the input for the internal model. A comparison of the two MPC strategies shows that, while inclusion of the wheel dynamics in the MPCt formulation results in a bigger optimization problem and requires for faster sampling times, no noticeable advantages in performance over the MPCs are observed. Another point to notice is that including both the longitudinal slip constraints and the torque constraints in the MPC formulation (something that was implemented in one way or another in both MPC strategies) gives a better knowledge of the system to the controller and results in more effective control actions.

Simulations in a high fidelity environment confirm the effectiveness of the controller in correcting terminal understeer behaviour and the importance of constraining both the state and the input of the system for improved stability. While similar trajectories are followed from both the MPCs and the LQR in the case of a U-turn scenario, the MPCs achieves this with much lower sideslip angle and yaw rate values. The importance of accounting for the system constraints becomes crucial in the case of a double-lane change scenario, where the MPCs keeps the vehicle stable by enforcing the state and input constraints. Finally, both scenarios show that the strategy is implementable in real time even when a generic QP solver is used.

\section{Funding}

This work is undertaken within the Evoque_e project, cofunded by the UKs innovation agency, Innovate UK. It is also supported by EPSRC under Grant EP/K502820/1. 


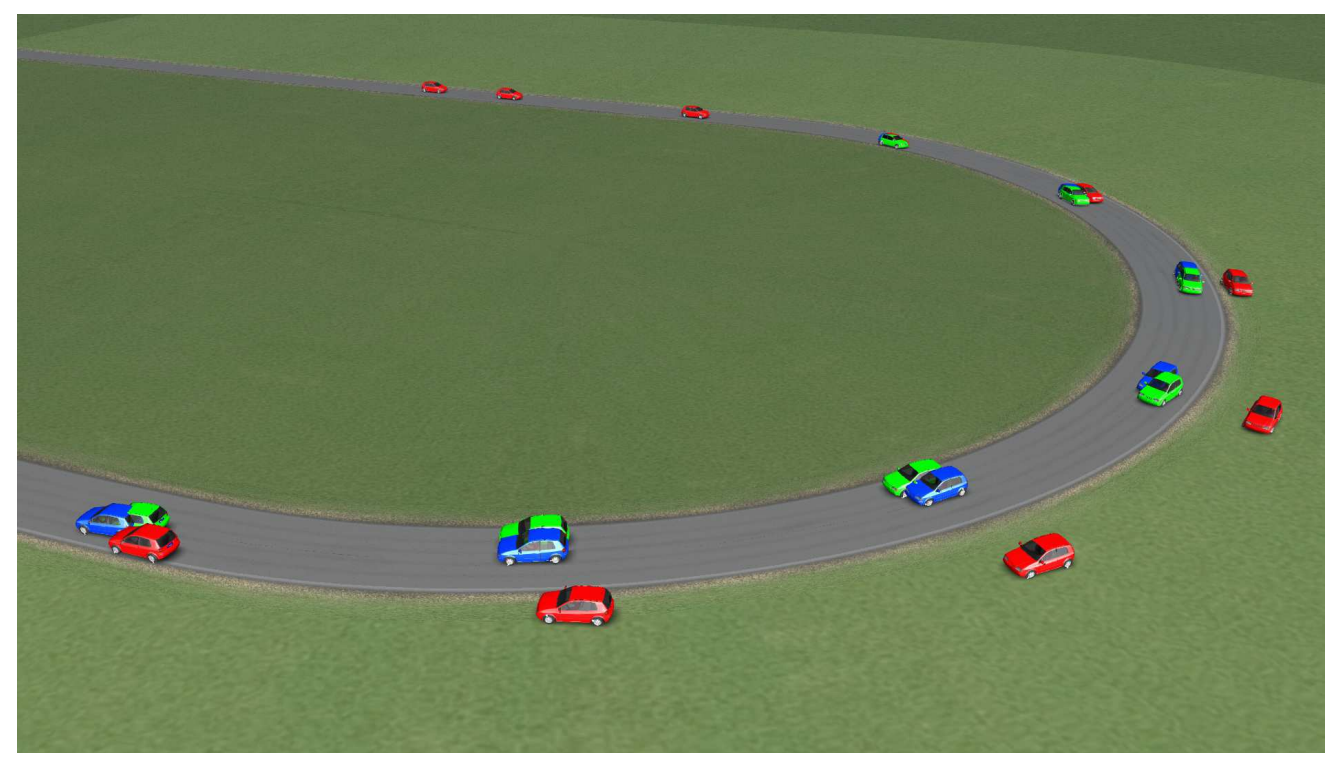

(a) Trajectory

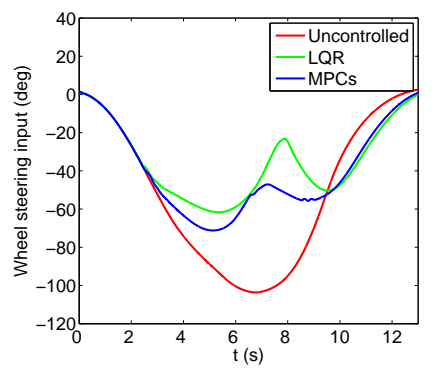

(b) Wheel steering input

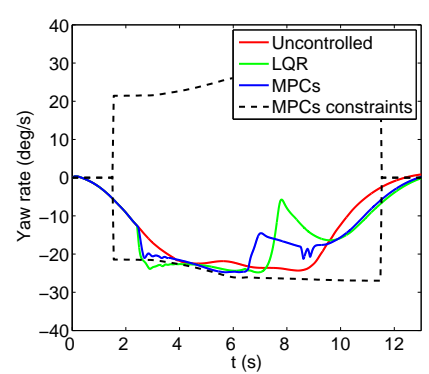

(e) Yaw rate

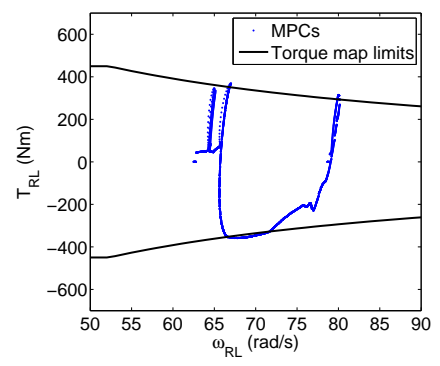

(h) Rear-left wheel torque

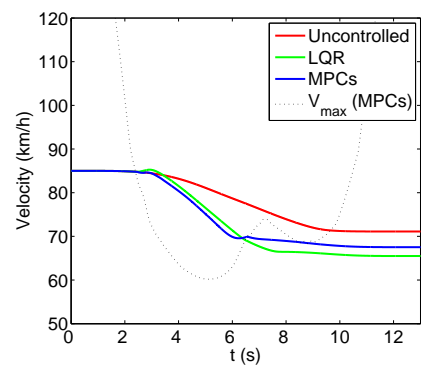

(c) Velocity

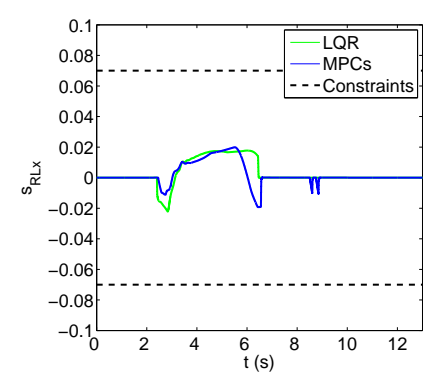

(f) Rear-left wheel long. slip

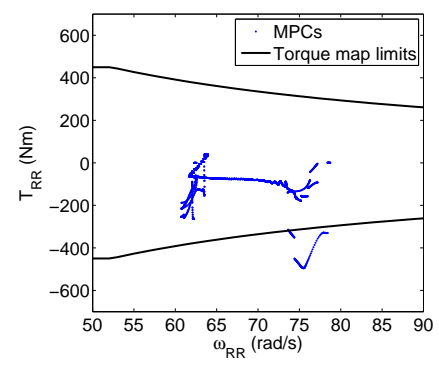

(i) Rear-right wheel torque

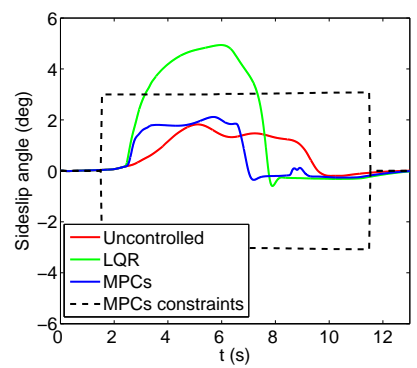

(d) Sideslip angle

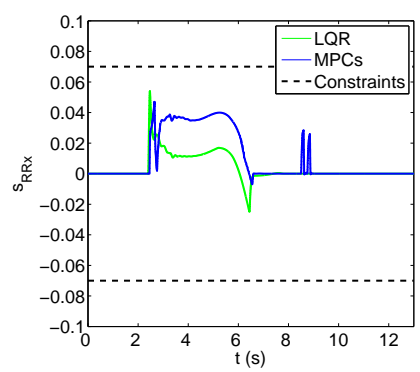

(g) Rear-right wheel long. slip

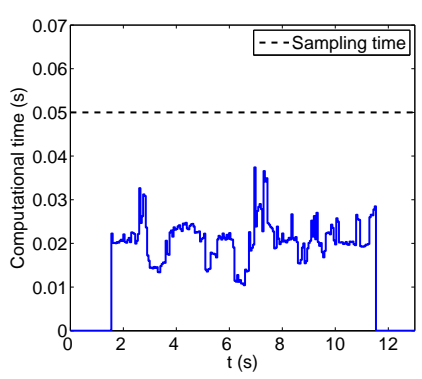

(j) Computational time

Figure 15. Both the MPCs and LQR achieve a tighter trajectory (a) while allowing for smaller steering inputs from the driver (b), but at the expense of large sideslip angle and yaw rate overshoots (d)-(e) for the LQR case. The rear wheel torque requests from the MPCs are largely kept within the torque map limits (h)-(i) while the computational time remains below the sampling time for the duration of the simulation $(\mathrm{j})$. 


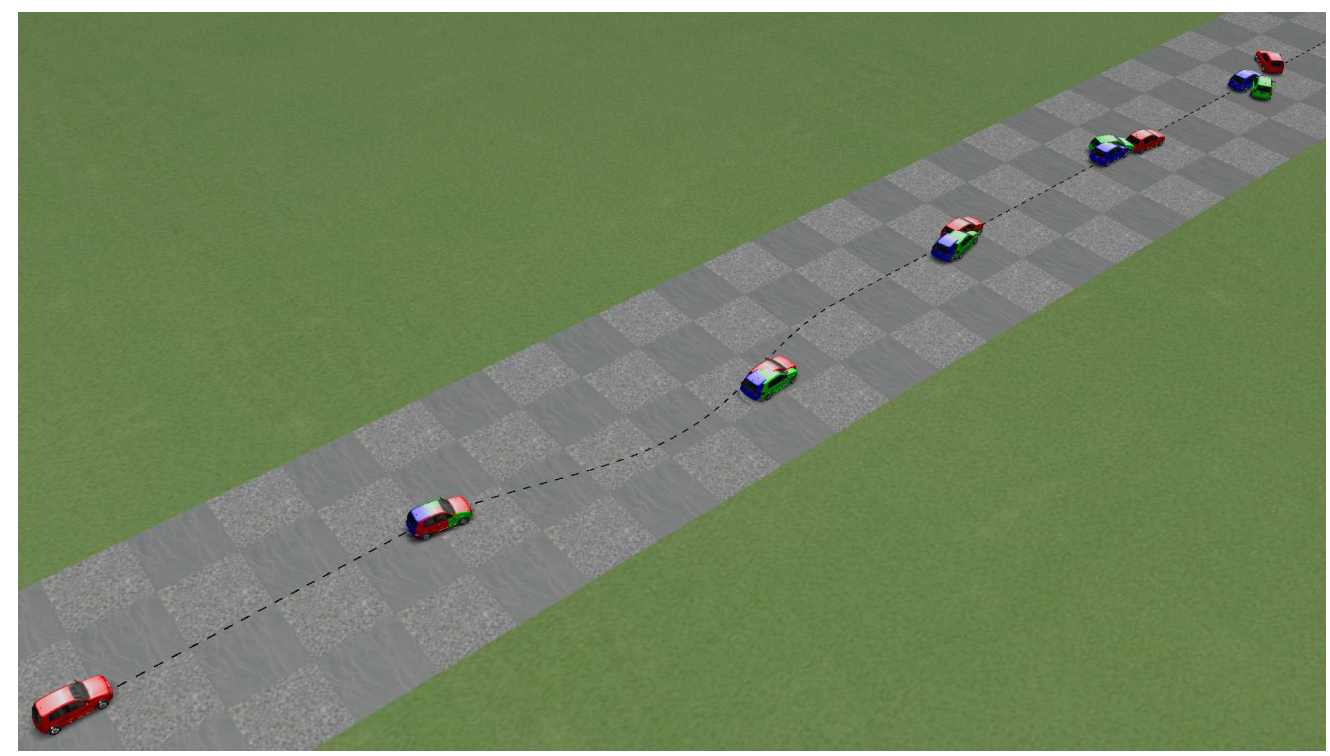

(a) Trajectory

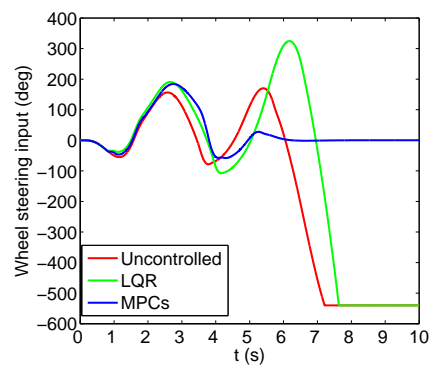

(b) Wheel steering input

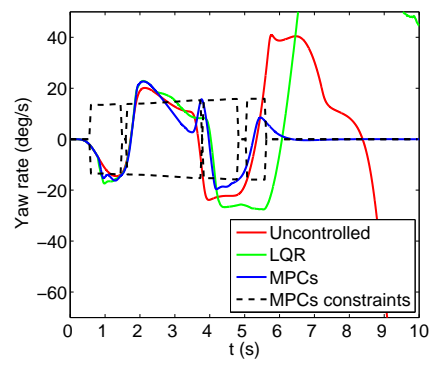

(e) Yaw rate

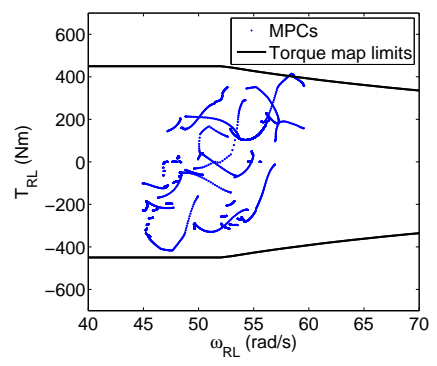

(h) Rear-left wheel torque

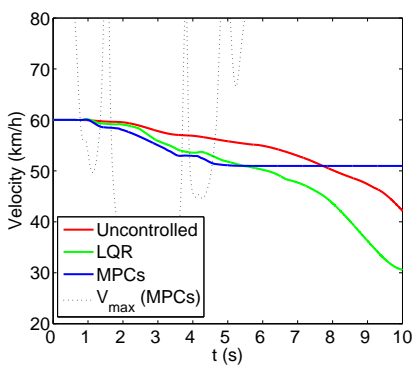

(c) Velocity

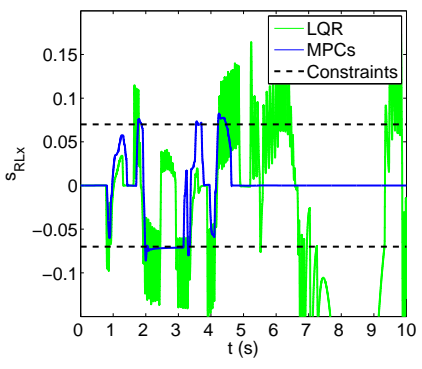

(f) Rear-left wheel long. slip

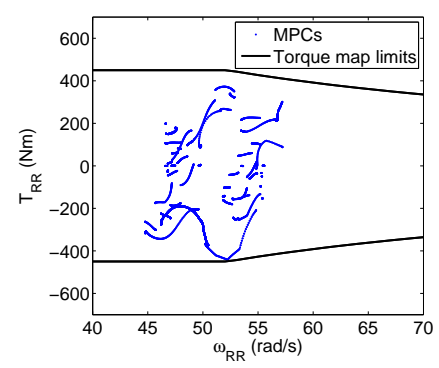

(i) Rear-right wheel torque

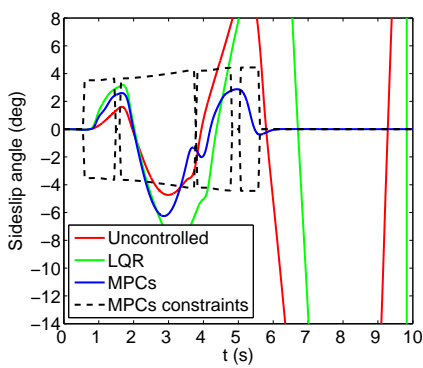

(d) Sideslip angle

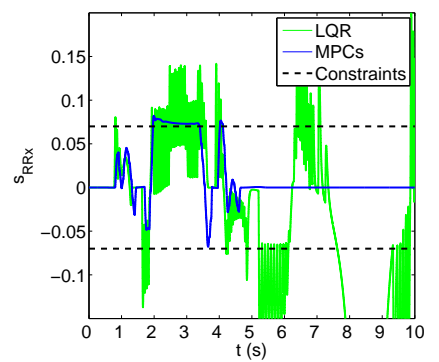

(g) Rear-right wheel long. slip

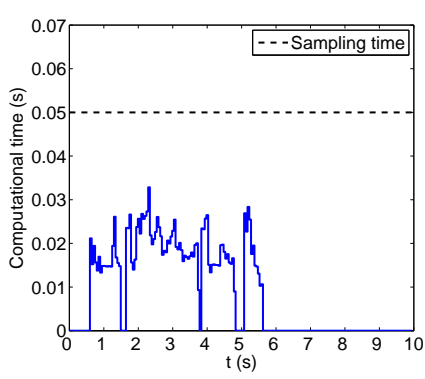

(j) Computational time

Figure 16. While the MPCs strategy successfully negotiates the double-lane change by enforcing the sideslip angle, yaw rate and tyre slip constraints (d)-(g), the LQR strategy can not keep the vehicle stable despite the switching strategy used in this case to keep the controller active only within the maximum longitudinal slip threshold (f)-(g). The rear wheel torque requests from the MPCs are largely kept within the torque map limits (h)-(i) while the computational time remains below the sampling time for the duration of the simulation (j). 


\section{References}

[1] Antonov S, Fehn A, Kugi A. Unscented Kalman Filter for vehicle state estimation. Veh Syst Dyn. 2011;49(9): 1497-1520.

[2] Astrom K, Wittenmark B. Computer-Controlled Systems. 2nd ed. Upper Saddle River (NJ): Prentice-Hall; 1997.

[3] Bakker E, Nyborg L, Pacejka HB. Tyre Modelling for Use in Vehicle Dynamics Studies. SAE Technical Paper 870421; 1987.

[4] Barbarisi O, Palmieri G, Scala S, Glielmo L. LTV-MPC for Yaw Rate Control and Side Slip Control with Dynamically Constrained Differential Braking. Eur J Control. 2009;15(3-4): 468-479.

[5] Beal CE, Gerdes JC. Model Predictive Control for Vehicle Stabilization at the Limits of Handling. IEEE Trans. Control Syst. Technol. 2013;21(4): 1258-1269.

[6] Canale M, Fagiano L. Vehicle yaw control using a fast NMPC approach. Proceedings of the 47th IEEE Conference on Decision and Control, Cancun, Mexico; 2008.

[7] Di Cairano S, Tseng HE, Bernardini D, Bemporad A. Vehicle Yaw Stability Control by Coordinated Active Front Steering and Differential Braking in the Tire Sideslip Angles Domain. IEEE Trans. Control Syst. Technol. 2013;21(4): 1236-1248.

[8] Falcone P, Ali M, Sjoberg J. Predictive Threat Assessment via Reachability Analysis and Set Invariance Theory. IEEE Trans. Intell. Transp. Syst. 2011;12(4): 1352-1361.

[9] Gillespie TD. Fundamentals of Vehicle Dynamics. Warrendale (PA): SAE International; 1992.

[10] Gray A, Ali M, Gao Y, Hedrick JK, Borrelli F. Integrated threat assessment and control design for roadway departure avoidance. Proceedings of the 15th IEEE Conference on Intelligent Transportation Systems, Anchorage, USA; 2012.

[11] Huh K, Hong D, Yoon P, Kang H, Hwang I. Robust Wheel-Slip Control for Brake-by-wire Systems. SAE Technical Paper 2005-01-1584; 2005.

[12] Kang J, Kyongsu Y, Heo H. Control Allocation based Optimal Torque Vectoring for 4WD Electric Vehicle. SAE Technical Paper 2012-01-0246; 2012.

[13] Khalil HK. Nonlinear Systems. 3rd ed. Upper Saddle River (NJ): Prentice-Hall; 2002.

[14] Kiencke U, Nielsen L. Automotive Control Systems: For Engine, Driveline, and Vehicle. Berlin: Springer; 2000.

[15] Kim J, Kim H. Electric Vehicle Yaw Rate Control using Independent In-Wheel Motor. Proceedings of the 4th IEEE Power Conversion Conference, Nagoya, Japan; 2007.

[16] Ko S, Ko J, Lee S, Cheon J, Kim H. Development of a vehicle stability control algorithm using velocity and yaw rate for an in-wheel drive vehicle. Proceedings of the 8th IEEE Vehicle Power and Propulsion Conference, Seoul, South Korea; 2012.

[17] Maciejowski JM. More Safety with Vehicle Stability Control. SAE Technical Paper 2007-01-2759; 2007.

[18] Kiencke U, Nielsen L. Predictive control with constraints. Upper Saddle River (NJ): Prentice Hall; 2002.

[19] Morari M, Garcia CE, Prett DM. Model predictive control: Theory and practice - A survey. Automatica. 1989;25(3): 335-348.

[20] Gill PE, Murray W, Wright MH. Practical Optimization. London: Academic Press; 1981.

[21] Rajamani R. Vehicle Dynamics and Control. 2nd ed. Berlin: Springer; 2012.

[22] Siampis E, Massaro M, Velenis E. Electric Rear Axle Torque Vectoring for Combined Yaw Stability and Velocity Control near the Limit of Handling. Proceedings of the 52nd IEEE Conference on Decision and Control, Florence, Italy; 2013.

[23] Siampis E, Velenis E, Longo S. Predictive Rear Wheel Torque Vectoring Control with Terminal Understeer Mitigation using Nonlinear Estimation. Proceedings of the 54th IEEE Conference on Decision and Control, Osaka, Japan; 2015, submitted.

[24] van Zanten A. Bosch ESP Systems: 5 Years of Experience. SAE Technical Paper 2000-01-1633; 2000.

[25] Velenis E, Frazzoli E, Tsiotras P. On Steady-State Cornering Equilibria for Wheeled Vehicles with Drift. Proceedings of the 48th IEEE Conference on Decision and Control, Shanghai, China; 2009.

[26] Velenis E, Katzourakis D, Frazzoli E, Tsiotras P, Happee R. Steady-State Drifting Stabilization of RWD Vehicles. Control Eng Pract. 2011;19(11): 1363-1376. 
2015-07-20

Rear wheel torque vectoring model predictive control with velocity regulation for electric vehicles

\section{Siampis, Efstathios}

Taylor and Francis

Efstathios Siampis, Efstathios Velenis and Stefano Longo. Rear wheel torque vectoring model predictive control with velocity regulation for electric vehicles. Vehicle System Dynamics:

International Journal of Vehicle Mechanics and Mobility. Volume 53, Issue 11, 2015. pp1555-1579 http://dx.doi.org/10.1080/00423114.2015.1064972

Downloaded from Cranfield Library Services E-Repository 